\title{
Structure and spectroscopy of phosphorus cluster anions: Theory (simulated annealing) and experiment (photoelectron detachment)
}

\author{
R. O. Jones, G. Ganteför, S. Hunsicker, and P. Pieperhoff \\ Institut für Festkörperforschung, Forschungszentrum Jülich, D-52425 Jülich, Germany
}

\begin{abstract}
Photoelectron detachment measurements have been performed on singly charged phosphorus cluster anions with up to nine atoms, generated by a pulsed arc cluster ion source (PACIS). Transitions between the anion ground states and states of the neutral clusters are observed for all clusters, and vibrational fin structure in both dimer and trimer. A comparison with the results of density functional calculations with simulated annealing - an extension to negative ions of earlier work on neutral and positively charged clusters - provides a consistent overall picture for all cluster sizes and the firs experimental structural information on several.
\end{abstract}

\section{INTRODUCTION}

The structural properties of phosphorus have provided a continuing challenge for many years. The bulk forms include several crystalline phases [puckered layers (black, orthorhombic), pentagonal tubes comprising $\mathrm{P}_{8}$ and $\mathrm{P}_{9}$ cages (Hittorf's violet, monoclinic), and a rhombohedral structure related to that of $\alpha$-arsenic] and a great variety of "red" amorphous structures. ${ }^{1}$ The structures of phosphorus clusters have been particularly difficul to characterize. Although experimental data on the dimer and the tetramer have been available for over 60 years, ${ }^{2}$ it is less than 10 years since $\mathrm{P}_{n}^{+}$ions were identifie mass spectrometrically up to $n=24 .{ }^{3}$ The dominant components in the vapor phase of the group Va (group 15) elements (P, As, $\mathrm{Sb}, \mathrm{Bi}$ ) are tetramers, and clusters with multiples of four atoms dominate in the mass spectra obtained, for example, in photoionization measurements of $\mathrm{Sb}_{n}$ with $n$ up to $300 .{ }^{4}$ Phosphorus clusters have often been studied as components of organometallic and other molecules. ${ }^{5,6}$ However, although there have been several theoretical studies of charged and neutral phosphorus clusters, and systematic trends in the structural patterns discussed by several authors, ${ }^{6-8}$ there has been an almost complete lack of experimental studies that provide structural information about the isolated clusters.

In the present study, we have used a pulsed arc cluster ion source (PACIS) (Ref. 9) to generate phosphorus cluster anions $\mathrm{P}_{n}^{-}$up to $n=9$, extending similar work performed recently on clusters of sulfur, the neighboring element of the periodic table. ${ }^{10}$ The use of laser photoelectron detachment ${ }^{11}$ enables us to study transitions from the ground state of $\mathrm{P}_{n}^{-}$to states of $\mathrm{P}_{n}$, and to observe vibrational fin structure in the dimer and trimer. To analyze the measurements on singly charged anions, we have extended earlier calculations in our institute on a range of forms of phosphorus. We use the density functional (DF) formalism, ${ }^{12}$ mainly with a local spin density (LSD) approximation for the exchange-correlation energy $E_{\mathrm{xc}}$, combined with molecular dynamics (MD). ${ }^{13}$ This approach has been applied to simulations of amorphous (red) phosphorus at $300 \mathrm{~K},{ }^{14}$ to study the polymerization of an assembly of $\mathrm{P}_{4}$ tetrahedra to a disordered network in the liquid, ${ }^{15}$ as well as to fin the stable structures of atomic clusters. ${ }^{16-18}$ In the present work, the structures so obtained have also been used as input for calculations with other schemes. We perform calculations for neutral clusters at the anionic and other geometries, and we have identifie new $\mathrm{P}_{n}$ isomers, including one for the heptamer that could be the most stable. We have also studied $\mathrm{P}_{8}^{2-}$, which has structures analogous to those of the dianion of cyclooctatetraene, the $10 \pi$-system $(\mathrm{CH})_{8}^{2-}$.

The earlier calculations on neutral and charged clusters with up to 11 atoms ${ }^{16-18}$ led to some unexpected predictions. The most stable isomers found for $\mathrm{P}_{5}, \mathrm{P}_{6}$, and $\mathrm{P}_{7}$ were derived from the "roof" or "butterfly' structure of $\mathrm{P}_{4}\left(C_{2 v}\right)$ by the addition of one, two, and three atoms, respectively, and for $\mathrm{P}_{8}$ a wedge-shaped $\left(C_{2 v}\right)$ structure related to the cuneane isomer of $(\mathrm{CH})_{8} \cdot{ }^{16}$ There are striking similarities in the bond angle distributions in amorphous phosphorus and in $\mathrm{P}_{n}$ clusters with up to 11 atoms. ${ }^{14}$ However, while structures of clusters and small molecules are given reliably by calculations using a LSD approximation for $E_{\mathrm{xc}}$, energy differences - such as dissociation and cohesive energies - are less reliable. ${ }^{12}$ Although subsequent calculations of $\mathrm{P}_{n}(n$ even) clusters up to $\mathrm{P}_{28}$ using correlated wave functions ${ }^{19}$ support the assignments of the ground states found in the LSD calculations for $\mathrm{P}_{6}$ and $\mathrm{P}_{8}$, they showed some differences in the ordering of the isomers and indicated that two $\mathrm{P}_{4}$ tetrahedra should be more stable than the cuneane isomer of $\mathrm{P}_{8}$. A study of non-local modification to the form of $E_{\mathrm{xc}}-$ in particular, the generalized gradient approximations (GGA) developed for the exchange energy by Becke ${ }^{20}$ and for the correlation energy by Perdew ${ }^{21}$ - led to significan improvements in the energy differences in both phosphorus and arsenic clusters, ${ }^{22}$ with only a modest increase in computing requirements. The effects of non-local modification to the LSD approximation are also studied in the present work.

Phosphorus cluster anions up to $n=9$ have been detected previously by Snodgrass et al., ${ }^{23}$ who also performed photoelectron spectroscopy on $\mathrm{P}_{2}^{-}$. Photoelectron spectroscopy on cluster anions of other group Va (group 15) elements has been carried out by Polak et al. ${ }^{24}$ (antimony $\mathrm{Sb}_{n}$ and bismuth clusters $\mathrm{Bi}_{n}$ to $\left.n=4\right)$ and by Gausa et al. ${ }^{25}\left(\mathrm{Sb}_{n}^{-}\right.$and $\mathrm{Bi}_{n}^{-}$to 
$n=9$, comparison with density functional calculations to $n=5)$. These and other works will be discussed below.

Experimental details are given in Sec. II A and the results in Sec. II B. The features of the method of calculation needed in the present context are described in Sec. III A, and we present the results in Sec. III B. The comparison of the present measurements and calculations (Sec. IV) focuses on the vertical detachment energies and the vibration frequencies. Our fina remarks are given in Sec. V.

\section{EXPERIMENT}

The pulsed arc cluster ion source has been described in detail elsewhere, ${ }^{9,26}$ and the present measurements are similar to recent work on sulfur clusters. ${ }^{10}$ The source used in that work has been modifie for operation with highly reactive materials such as phosphorus and the alkali and rare earth metals. We now summarize the experimental arrangement and discuss those aspects specifi to phosphorus.

\section{A. Apparatus}

The phosphorus clusters are generated by an electric arc, the lower electrode of which is made from copper in the form of a crucible containing the element. The arc burns between this electrode and an upper electrode consisting of the tip of a molybdenum rod. The gap between the electrodes can be adjusted from the outside during operation, which is essential to optimize the output of anions. After ignition of the arc by a high voltage pulse $(450 \mathrm{~V})$, a voltage of $150 \mathrm{~V}$ is applied for $50 \mu \mathrm{s}$ using a $1 \Omega$ resistor that limits the current to approximately $100 \AA$. These parameters are material dependent and the peak current for phosphorus is relatively low (for silicon the maximum current applied is $5000 \mathrm{~A}$ ).

The source chamber is designed as a glove box and contains a reservoir of the material. To refil the source from the reservoir we floo the chamber with an inert gas $\left(\mathrm{N}_{2}\right)$ and use two gloves that are introduced into the chamber through separate valves. The gloves are mounted on the high pressure side of the valves, and the inside surfaces are not in contact with air. The source is cleaned and maintained using the same procedure. To evacuate the chamber, the gloves are withdrawn and the valves closed. Generation of bare clusters of reactive materials requires cooling the source to low temperatures, a method firs used by Honea et al. ${ }^{27}$ to produce $\mathrm{Na}_{n}$ clusters in a laser vaporization source cooled by liquid nitrogen (LN2). In the PACIS the lower electrode containing the phosphorus is connected to a LN2 reservoir.

The most common form of red phosphorus is a fin powder that tends to be blown away by the helium pulses. The spectra shown below were obtained using red phosphorus pieces (1-5 $\mathrm{mm}$ in diameter) obtained as a byproduct of the industrial production of the powder (Hoechst AG, Hürth, Germany). The samples are not well characterized, but probably comprise the red (amorphous), white, and violet modification of the element. Outside the chamber the samples must be stored under water, which is a source of oxide and hydride impurities in spite of the efforts described above to minimize contamination. Without the glovebox and LN2 cooling, however, $\mathrm{P}_{2}^{-}$was the only bare phosphorus cluster anion that could be detected.

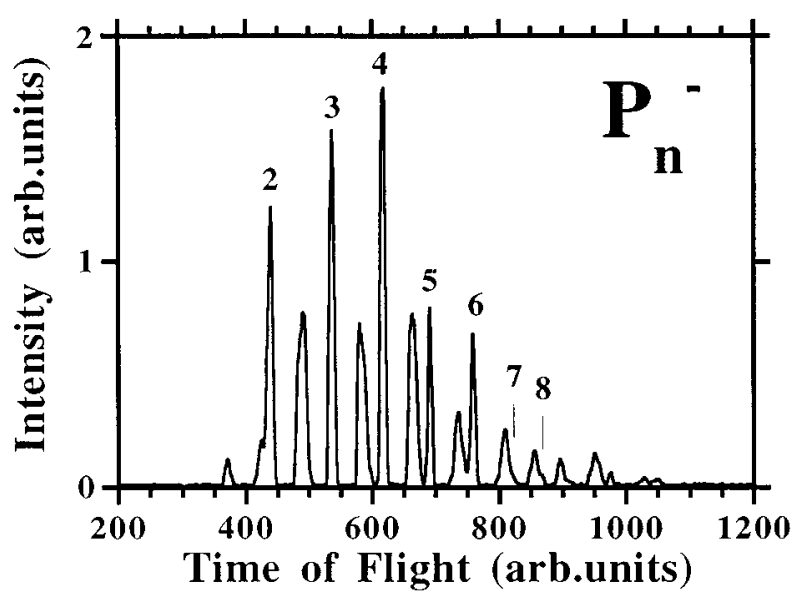

FIG. 1. Mass spectrum.

A pulse of He gas is flushe through the gap between the electrodes during ignition and carries the $\mathrm{He} / \mathrm{P}$ plasma into an extender, where phosphorus clusters grow on cooling. After leaving the extender, the gas containing the neutral and ionic clusters passes through a conical nozzle into vacuum and forms a supersonic jet. After passing a skimmer, the anions are accelerated in a pulsed electric fiel (WileyMcLaren time-of-fligh (ToF) mass spectrometer), and a typical mass spectrum (Fig. 1) contains lines corresponding to phosphorus clusters with up to 8 atoms. The overall shape of the spectrum depends on the source parameters and is optimized in Fig. 1 for $n=2-4$.

In addition to peaks at the masses of the bare $\mathrm{P}_{n}^{-}$clusters, we observe broader features that are assigned to $\mathrm{P}_{n} \mathrm{O}_{m}^{-}$ and $\mathrm{P}_{n} \mathrm{OH}_{m}^{-}$clusters. The larger width can be explained by a varying number of hydrogen atoms, and the corresponding peaks cannot be resolved due to the limited mass resolution. It is possible that $\mathrm{P}_{n} \mathrm{O}_{2}^{-}$and $\mathrm{P}_{n} \mathrm{O}_{2} \mathrm{H}_{m}^{-}$clusters contribute to the intensity of features assigned to the bare $\mathrm{P}_{n}^{-}$clusters, since the mass of $\mathrm{O}_{2}(32.00 \mathrm{amu})$ is similar to the mass of the phosphorus atom (30.97 amu). However, the photoelectron spectra provide a much more sensitive method for identifying bare and compound clusters. The reproducibility and the shape of the spectra shown below indicate that contributions from impurities to the photoelectron spectra are very small.

Finally, the anion beam is directed into the interaction region of a magnetic bottle ToF electron spectrometer. A selected bunch of phosphorus clusters of a particular size is irradiated by a laser pulse of energy $100 \mu \mathrm{J}-1 \mathrm{~mJ}$ (second, third, and fourth harmonics of a Nd-Yag laser, corresponding to $h \nu=2.33,3.49$, and $4.66 \mathrm{eV}$, respectively). The detached electrons are guided towards the electron detector by magnetic fields and their kinetic energies are determined from the measured time-of-flight The energy resolution, which is limited by the Doppler effect due to the velocity of the anions, increases with decreasing electron kinetic energy and increasing mass of the anions. In the present measurements it varies between $20-100 \mathrm{meV}$. 


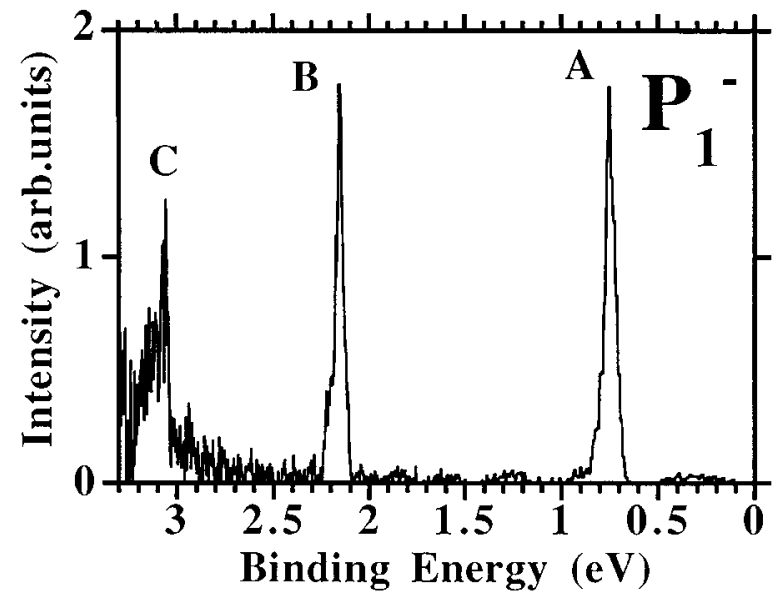

FIG. 2. Photoelectron spectra of $\mathrm{P}_{1}^{-}$recorded at $h \nu=3.49 \mathrm{eV}$ photon energy.

\section{B. Results}

Figs. 2-6 show photoelectron spectra of the $\mathrm{P}_{n}^{-}$clusters recorded at photon energies $h \nu=2.33 \mathrm{eV}$ (Figs. 3,6), $3.49 \mathrm{eV}$ (Figs. 2, 4) and $4.66 \mathrm{eV}$ (Fig. 5). In general, the signal-to-

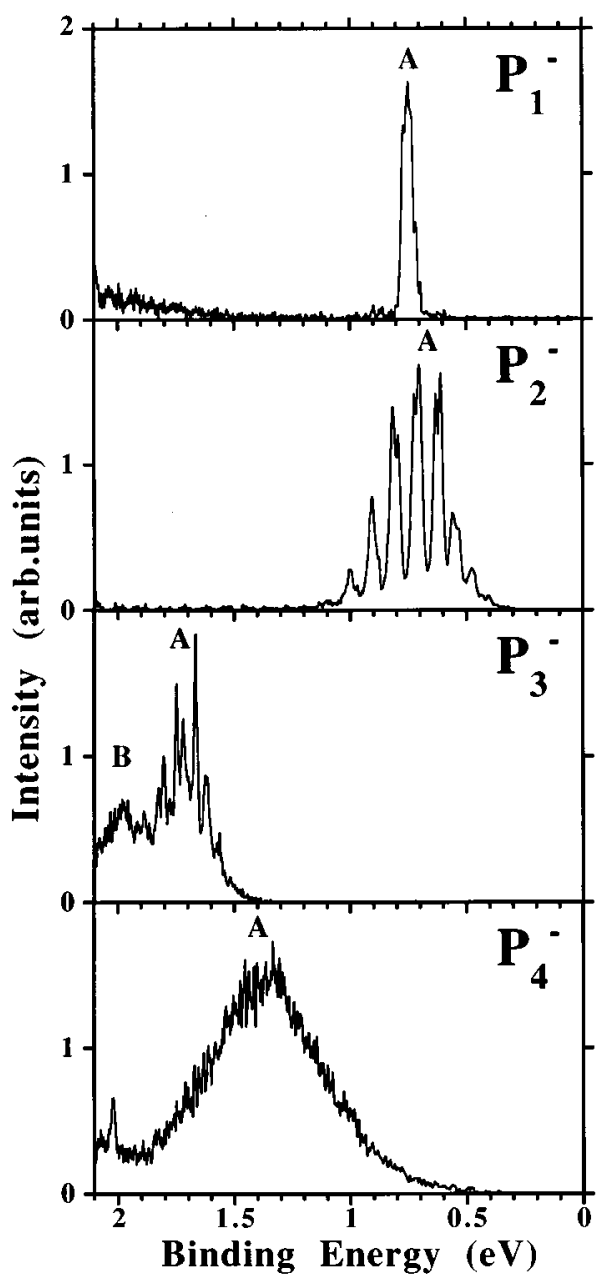

FIG. 3. Photoelectron spectra of $\mathrm{P}_{n}^{-}$-clusters $(n=1-4)$ recorded at $h \nu=2.33 \mathrm{eV}$ photon energy. See text for a discussion of the features marked.

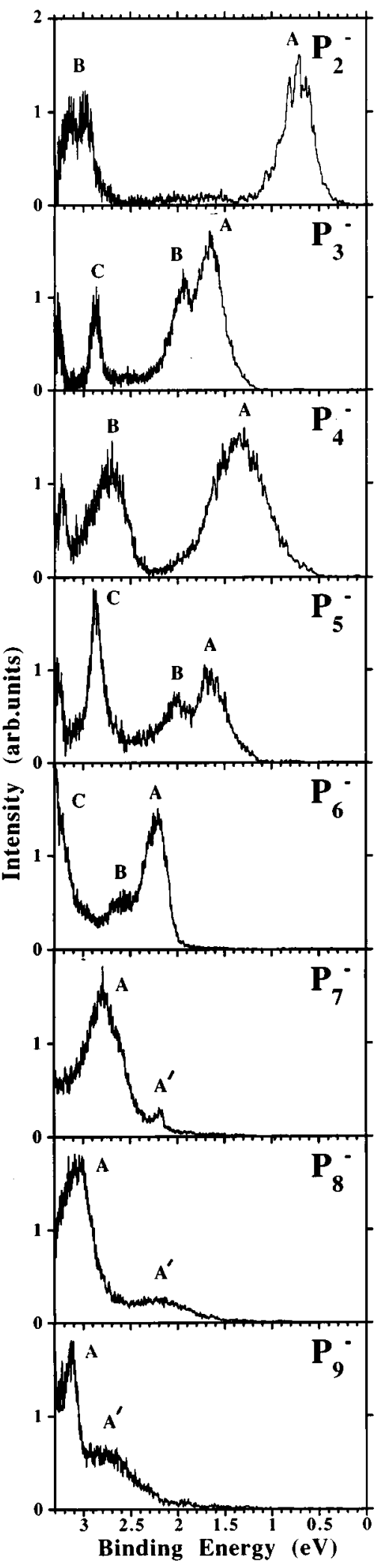

FIG. 4. Photoelectron spectra of $\mathrm{P}_{n}^{-}$-clusters $(n=2-9)$ recorded at $h \nu=3.49 \mathrm{eV}$ photon energy. See text for a discussion of the features marked.

noise ratio of the spectra is lower than for $\mathrm{S}_{n}^{-}$clusters, ${ }^{10}$ because the anion intensity generated by the PACIS is about an order of magnitude less. The spectra at the three photon energies have many features in common, and transitions into 


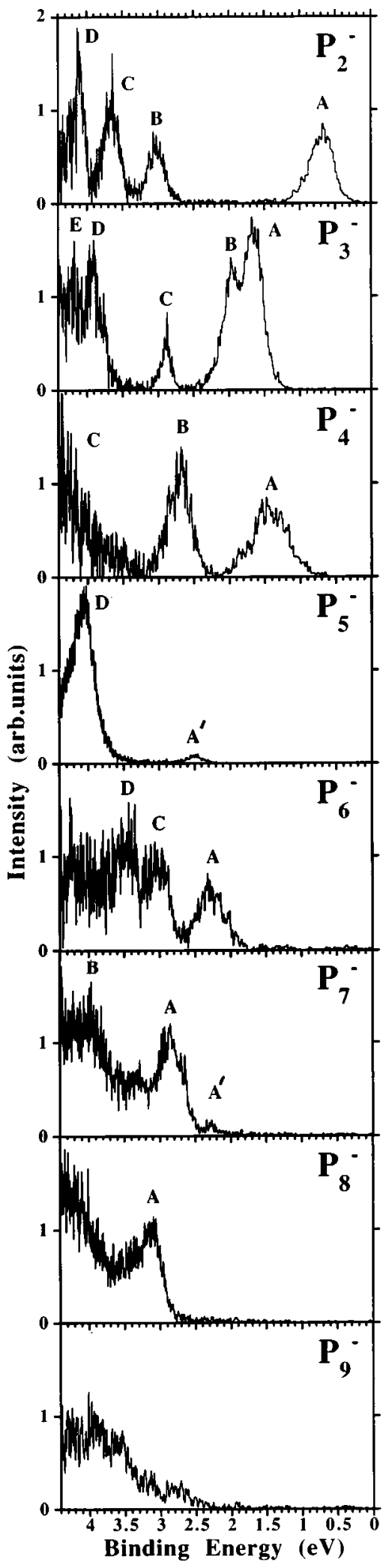

FIG. 5. Photoelectron spectra of $\mathrm{P}_{n}^{-}$-clusters $(n=2-9)$ recorded at $h \nu=4.66 \mathrm{eV}$ photon energy. See text for a discussion of the features marked.

higher-lying excited states of the neutral clusters are evident. Some spectra at $h \nu=3.49 \mathrm{eV}$ show features at kinetic energies below $0.3 \mathrm{eV}$ that do not occur at the corresponding binding energies $(\mathrm{BE})$ in the $h \nu=4.66 \mathrm{eV}$ spectra. We as-

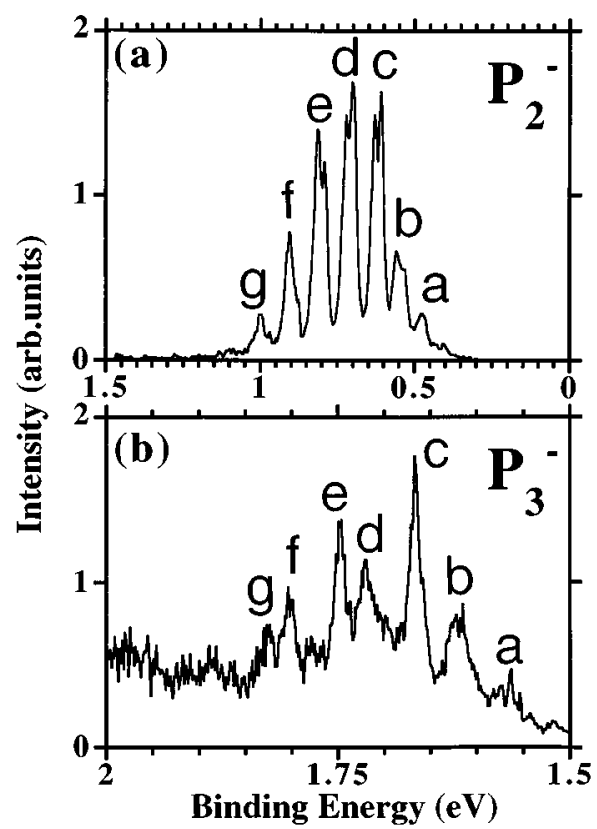

FIG. 6. Photoelectron spectra of (a) $\mathrm{P}_{2}^{-}$and (b) $\mathrm{P}_{3}^{-}$, for $h \nu=2.33 \mathrm{eV}$ photon energy. Note the difference in the energy scales.

sume that these features arise from experimental limitations at very low kinetic energies.

The resolution is best at low photon energy, because the electron kinetic energy is lower, and the spectra of $\mathrm{P}_{2}^{-}$and $\mathrm{P}_{3}^{-}$show vibrational fin structure (Fig. 6). The VDE is taken as the $\mathrm{BE}$ of the maximum of the peak assigned to the transition from the electronic ground state of the anion into the electronic ground state of the neutral cluster, which is not necessarily the feature at lowest BE (see below). The shapes of the peaks in the spectra provide useful additional information in several cases. The VDE's and the BE's of the observed features are listed in Table I.

\section{1. $P_{1}^{-}$}

Fig. 2 shows the spectrum of $\mathrm{P}_{1}^{-}$recorded at $h \nu=3.49$ $\mathrm{eV}$. The poor signal-to-noise ratio reflect the low relative intensity of the atom in the mass spectrum. The electron affinit of phosphorus (also the ionization energy of $\mathrm{P}_{1}^{-}$) is

TABLE I. The BE's in eV of the electronic transitions observed in the photoelectron spectra of $\mathrm{P}_{n}^{-}$. The uncertainty in the measurements is $\pm 0.05 \mathrm{eV}$ except for $n=1$. The BE's of features A correspond to the VDE's.

\begin{tabular}{ccccccc}
\hline \hline$n$ & $\mathrm{~A}^{\prime}$ & $\mathrm{A}$ & $\mathrm{B}$ & $\mathrm{C}$ & $\mathrm{D}$ & $\mathrm{E}$ \\
\hline 1 & & 0.75 & 2.16 & 3.07 & & \\
2 & & 0.68 & 3.03 & 3.65 & 4.10 & \\
3 & & 1.68 & 1.96 & 2.89 & 3.91 & 4.32 \\
4 & & 1.35 & 2.69 & $>4.40$ & & \\
5 & 2.49 & 4.04 & & & & \\
6 & & 2.22 & 2.59 & 3.00 & 3.47 & \\
7 & 2.19 & 2.79 & 4.00 & & & \\
8 & 2.20 & 3.05 & & & & \\
9 & 2.70 & 3.11 & & & & \\
\hline \hline
\end{tabular}


$0.7465 \mathrm{eV}^{28}$ and the lowest excited states of the atom are $1.41 \mathrm{eV}\left({ }^{2} D_{0}\right)$ and $2.32 \mathrm{eV}\left({ }^{2} P_{0}\right)$ above the atomic ground state $\left({ }^{4} S_{0}\right) \cdot{ }^{29}$ The photoelectron spectrum of $\mathrm{P}_{1}^{-}$shows these three peaks clearly, although the spin-orbit splitting is not resolved in the present experiment.

\section{2. $P_{2}^{-}$}

The photoelectron spectra of $\mathrm{P}_{2}^{-}$show four electronic transitions (Figs. 3-6, A-D). Feature $\mathrm{A}$ is assigned to the ground state transition with VDE $0.68 \pm 0.05 \mathrm{eV}$. In agreement with earlier work ${ }^{23}$ vibrational fin structure is resolved at low photon energy (Figs. 3,6(a)). Seven single vibrational transitions $(\mathrm{a}-\mathrm{g})$ are resolved, with a pronounced increase in intensity from $b$ to $c$. We assign feature $c$ to the $0-0$ transition corresponding to an adiabatic electron affinit of $\mathrm{P}_{2}^{-}$of 0.63 $\pm 0.05 \mathrm{eV}$. The spacing between the peaks c-g corresponds to the vibration frequency of $\mathrm{P}_{2}$ of $90 \pm 10 \mathrm{meV}$. Features a and $\mathrm{b}$ are assigned to hot band transitions $(0 \leftarrow 1 ; 0 \leftarrow 2)$ corresponding to a vibration frequency of $\mathrm{P}_{2}^{-}$of $70 \pm 15 \mathrm{meV}$. All features show a double peak with a splitting of $20 \pm 4 \mathrm{meV}$. This is close to the estimated spin-orbit splitting of the electronic ground state of $\mathrm{P}_{2}^{-30}$ and may reflec the presence in the beam of $\mathrm{P}_{2}^{-}$molecules of two states split by spin-orbit coupling. If we assume that the electronic temperature is high compared with this splitting $(20 \pm 4 \mathrm{meV})$, anions with both electronic states should be present in the beam with approximately equal intensity. The vibrational temperature can be estimated from the intensities of the hot bands (see Sec. II B 3.) to be $580 \pm 100 \mathrm{~K}$, if we assume that the Franck-Condon factors are equal for different transitions. This temperature is rather high, since anions generated by a PACIS or a laser vaporization source generally have temperatures $\sim 250-400 \mathrm{~K}$, depending on the source parameters. ${ }^{9}$

The peak positions and vibration frequencies differ from those determined by Snodgrass et al. (electron affinit 0.589 $\pm 0.025 \mathrm{eV} ; \operatorname{VDE} 0.684 \pm 0.025 \mathrm{eV} ; \omega_{e}\left(\mathrm{P}_{2}\right) 96 \pm 6 \mathrm{meV}$; $\left.\omega_{e}\left(\mathrm{P}_{2}^{-}\right) 78 \pm 6 \mathrm{meV}\right) .^{23}$ Part of the uncertainty arises from contamination of the surfaces of the apparatus when operated with a reactive and non-conducting material, which requires a daily recalibration of the spectrometer. Furthermore, we use atomic lines $\left(\mathrm{P}^{-}, \mathrm{Au}^{-}, \mathrm{Ag}^{-}\right)$that allow for reliable calibration in the binding energy range $1-4 \mathrm{eV}$, where several atomic transitions are available. The energy uncertainty is greater for smaller BE values. The earlier work ${ }^{23}$ showed only single peaks, which might be due to a higher rotation temperature.

\section{3. $P_{3}^{-}$}

The photoelectron spectra of $\mathrm{P}_{3}^{-}$display fiv electronic transitions (Figs. 3-6, A-E). Feature $\mathrm{A}$ is assigned to the ground state transition (VDE $1.68 \pm 0.05 \mathrm{eV}$ ) and exhibits vibrational fin structure (Fig. 6(b)) when recorded with $h \nu=2.33 \mathrm{eV}$. The BE's of the seven vibrational transitions identifie $(\mathrm{a}-\mathrm{g})$ are listed in Table III, and the intensity increases from $\mathrm{b}$ to $\mathrm{c}$ as in the dimer. We assign peak c tentatively to the $0 \leftarrow 0$ transition, corresponding to an adiabatic electron affinit of $1.665 \pm 0.05 \mathrm{eV}$. The constant spacing be-
TABLE II. Binding energies in $\mathrm{eV}$ of the vibrational transitions of features $\mathrm{a}-\mathrm{g}$ in the spectrum of $\mathrm{P}_{2}^{-}$(Fig. 6(a)). The two values (BE1, BE2) reflec the splitting of each peak, and the BE2 series is assigned to the ground state transition. The $\mathrm{BE}$ uncertainty is $\pm 0.05 \mathrm{eV}$. The assignments (assg.) to vibrational transitions from $\mathrm{P}_{2}^{-}$to the ground state of $\mathrm{P}_{2}$ are also given.

\begin{tabular}{cccccccc}
\hline \hline Peak & $\mathrm{a}$ & $\mathrm{b}$ & $\mathrm{c}$ & $\mathrm{d}$ & $\mathrm{e}$ & $\mathrm{f}$ & $\mathrm{g}$ \\
\hline assg. & $0 \leftarrow 2$ & $0 \leftarrow 1$ & $0 \leftarrow 0$ & $1 \leftarrow 0$ & $2 \leftarrow 0$ & $3 \leftarrow 0$ & $4 \leftarrow 0$ \\
BE1 & 0.47 & 0.54 & 0.61 & 0.70 & 0.79 & 0.88 & 0.98 \\
BE2 & 0.49 & 0.56 & 0.63 & 0.72 & 0.81 & 0.91 & 1.00 \\
\hline \hline
\end{tabular}

tween peaks c-e-g is assigned to the excitation of one high energy mode of $\mathrm{P}_{3}$ with a frequency of $80 \pm 7 \mathrm{meV}(0 \leftarrow 0$, $1 \leftarrow 0,2 \leftarrow 0)$. Feature $\mathrm{d}$ can be associated with the excitation of another mode of $\mathrm{P}_{3}$ with a frequency of $57 \pm 8 \mathrm{meV}(0$, $1 \leftarrow 0$ ), and peak $\mathrm{f}$ with the simultaneous excitation by a single photon of two modes $137 \mathrm{meV}$ above the $0 \leftarrow 0$ transition (assignment $1,1 \leftarrow 0$ ). Features $\mathrm{a}$ and $\mathrm{b}$ are assigned to hot band transitions and are significantl broader than peak c. The broadening may arise from unresolved fin structure, such as two thermally excited modes in $\mathrm{P}_{3}^{-}$that are very close in energy, and the average is given in Table II. Our spectrum is then consistent with the excitation of either two vibrational modes of $\mathrm{P}_{3}^{-}$(frequencies 42 and $54 \mathrm{meV}$ ) or a single mode (frequency $48 \pm 6 \mathrm{meV}$ ).

The spectrum measured for $\mathrm{Sb}_{3}^{-}$(Ref. 24) is very similar to that of $\mathrm{P}_{3}^{-}$, except that feature $\mathrm{C}$ is absent. Two peaks split by $0.16 \mathrm{eV}$ occur at low $\mathrm{BE}$ and another peak is found at 3.3 $\mathrm{eV}$.

\section{4. $P_{4}^{-}$}

The photoelectron spectra of $\mathrm{P}_{4}^{-}$display 3 electronic transitions (Figs. $3-5$, denoted $\mathrm{A}-\mathrm{C}$ ). Peak $\mathrm{A}$, which is assigned to the ground state transition (VDE $1.35 \pm 0.05 \mathrm{eV}$ ), is very broad (full width at half maximum of $0.35 \mathrm{eV}$ ), indicating a large difference between the ground state geometries of $\mathrm{P}_{4}^{-}$and $\mathrm{P}_{4}$. Feature $\mathrm{C}$ is only observed as an increase of emission signal at high $\mathrm{BE}$ in the $4.66 \mathrm{eV}$ spectrum, so that the measured $\mathrm{BE}$ is probably a lower bound. The spectrum of $\mathrm{P}_{4}^{-}$is strikingly similar to that of $\mathrm{Sb}_{4}^{-},{ }^{24}$ indicating that the electronic and geometric structures of these tetramers are very similar.

\section{5. $\mathrm{P}_{5}^{-}$}

The photoelectron spectra of $\mathrm{P}_{5}^{-}$for $h \nu=3.49 \mathrm{eV}$ (Fig. 4) and $4.66 \mathrm{eV}$ (Fig. 5) are completely different. The features observed in the former $(\mathrm{A}, \mathrm{B}, \mathrm{C})$ are located at almost the same BE's as the corresponding features in the spectrum of

TABLE III. Binding energies in $\mathrm{eV}$ of the vibrational transitions of features $\mathrm{a}-\mathrm{g}$ in the spectrum of $\mathrm{P}_{3}^{-}$(Fig. 6(b)). The uncertainty is $\pm 0.05 \mathrm{eV}$. The assignments (assg.) to vibrational transitions from the anion to the neutral electronic ground state are also given.

\begin{tabular}{cccccccc}
\hline \hline Peak & $\mathrm{a}$ & $\mathrm{b}$ & $\mathrm{c}$ & $\mathrm{d}$ & $\mathrm{e}$ & $\mathrm{f}$ & $\mathrm{g}$ \\
\hline assg. & $0 \leftarrow 2$ & $0 \leftarrow 1$ & $0 \leftarrow 0$ & $0,1 \leftarrow 0$ & $1,0 \leftarrow 0$ & $1,1 \leftarrow 0$ & $2,0 \leftarrow 0$ \\
$\mathrm{BE}$ & 1.567 & 1.618 & 1.665 & 1.721 & 1.745 & 1.803 & 1.825 \\
\hline \hline
\end{tabular}


$\mathrm{P}_{3}^{-}$(Fig. 4). They are slightly broader, and peak $\mathrm{C}$ is relatively about twice as intense as in the trimer spectrum. We assign the observed peaks to $\mathrm{P}_{3}^{-}$that is generated by a photofragmentation process:

$$
\begin{aligned}
& \mathrm{P}_{5}^{-}+h \nu \rightarrow \mathrm{P}_{3}^{-}+\mathrm{P}_{2} \\
& \mathrm{P}_{3}^{-}+h \nu \rightarrow \mathrm{P}_{3}+e^{-} .
\end{aligned}
$$

A strong photofragmentation signal indicates that the electron affinit of the cluster is higher than the photon energy used. ${ }^{31}$ The photoelectrons are generated in a twophoton process, with the firs photon inducing the fragmentation process, and the broader features are consistent with the high temperatures associated with photofragments. The change in the relative intensity of feature $\mathrm{C}$ is not an effect of temperature, because the integrated intensity of a peak corresponding to a single electronic transition depends on a matrix element that is independent of temperature. It is possible that the peaks $(\mathrm{A}, \mathrm{B})$ and $\mathrm{C}$ are associated with different isomers with very similar stabilities, since the relative intensities change little with changing source conditions. However, generation by a fragmentation process could result in a different isomer distribution.

The spectrum of $\mathrm{P}_{5}^{-}$recorded with a photon energy of $4.66 \mathrm{eV}$ (Fig. 5) is consistent with a high electron affinit . A strong feature $(\mathrm{A}, \mathrm{BE} 4.04 \mathrm{eV})$ is assigned to the ground state transition, and there is a weak peak $\left(\mathrm{A}^{\prime}\right)$ at lower $\mathrm{BE}$ of unknown origin. A similar spectrum has been recorded for $\mathrm{Sb}_{5}^{-}$by Polak et al. ${ }^{24}$ This molecule also has a very high $\operatorname{VDE}(\geqslant 3.8 \mathrm{eV})$ and a weak feature at lower BE $(\sim 2.0 \mathrm{eV})$, although no conclusive interpretation of the latter was given.

\section{6. $P_{6}^{-}$}

The photoelectron spectra of $\mathrm{P}_{6}^{-}$recorded at $h \nu=3.49$ eV (Fig. 4) and $4.66 \mathrm{eV}$ (Fig. 5) show four electronic transitions $\mathrm{A}-\mathrm{D}$, whose relative intensities depend strongly on the photon energy due to cross section effects. A and B are not resolved in Fig. 5. There is a structureless emission signal at high $\mathrm{BE}(3.5-4.5 \mathrm{eV})$ due possibly to overlapping electronic transitions.

\section{7. $P_{7}^{-}$}

Three electronic transitions $\left(\mathrm{A}^{\prime}, \mathrm{A}, \mathrm{B}\right)$ can be resolved in the photoelectron spectra of $\mathrm{P}_{7}^{-}$(Figs. 4,5). Feature $\mathrm{A}^{\prime}$ is weak, and its location close to the $\mathrm{BE}$ of peak $\mathrm{A}$ in the spectrum of $\mathrm{P}_{6}^{-}$suggests that it may originate from the photofragmentation process:

$$
\begin{aligned}
& \mathrm{P}_{7}^{-}+h \nu \rightarrow \mathrm{P}_{6}^{-}+\mathrm{P} \\
& \mathrm{P}_{6}^{-}+h \nu \rightarrow \mathrm{P}_{6}+e^{-} .
\end{aligned}
$$

Since the photon energy is far above the electron affinit, the emission signal due to fragmentation is weaker than the emission from the parent cluster. Feature A (VDE $2.79 \mathrm{eV}$ ) is assigned to the ground state transition. It shows a slight asymmetry with a shoulder at low $\mathrm{BE}(\sim 2.6 \mathrm{eV})$ and might comprise two unresolved peaks. However, the signal-tonoise ratio does not allow a quantitative analysis. There is a continuous, relatively structureless emission signal for BE's above $3 \mathrm{eV}$ and a further intense maximum $\mathrm{B}$ with $\mathrm{BE}$ $\sim 4.0 \mathrm{eV}$.

\section{8. $P_{8}^{-}$}

The single pronounced peak (A) in the spectra of $\mathrm{P}_{8}^{-}$ (Figs. 4,5) is assigned to the ground state transition (VDE $3.05 \mathrm{eV})$. There is a broad, weak feature in the $h \nu=3.49 \mathrm{eV}$ spectrum $\left(\mathrm{A}^{\prime}, \mathrm{BE} \sim 2.2 \mathrm{eV}\right)$ that is not observed in the 4.66 $\mathrm{eV}$ spectrum. The origin of this feature, an almost structureless emission signal that increases monotonically from $\mathrm{BE}$ $\sim 3 \mathrm{eV}$ towards higher energy, is unclear.

\section{9. $P_{9}^{-}$}

The spectrum for $h \nu=3.49 \mathrm{eV}$ (Fig. 4) is similar to that of $\mathrm{P}_{8}^{-}$with a pronounced peak (A, VDE $3.11 \mathrm{eV}$ ) assigned to the ground state transition. We neglect in the following a broad and weak feature $\left(\mathrm{A}^{\prime}\right)$ at lower $\mathrm{BE}$. The relative intensity of the anions drops above $n=8$, and the spectrum of $\mathrm{P}_{9}^{-}$at $h \nu=4.66 \mathrm{eV}$ is weak and featureless.

\section{CALCULATIONS}

\section{A. Computational details}

Details of the application of the MD/DF method to neutral $\mathrm{P}_{n}$ clusters have been given elsewhere, ${ }^{16}$ and the structures and binding energies calculated for the neutral clusters using the LSD approximation for $E_{\mathrm{xc}}$ are given in Refs. 16 and 18 . The calculations adopt periodic boundary conditions (PBC) with a large (f.c.c.) unit cell with lattice constant 30 a.u., a plane wave basis set with energy cutoff 5.3 a.u., and a single point $(k=0)$ in the Brillouin zone. The electron-ion interaction is described by the non-local pseudopotential of Bachelet et al., ${ }^{32}$ using the $d$-component of the potential as the reference local part ("s $p$-nonlocality"). The reader is referred to Refs. 16 and 18 for details and figure of all the $\mathrm{P}_{n}$-isomers calculated.

In the present work, as in the study of sulfur clusters, ${ }^{10}$ we focus on the cluster geometries and the energy differences between states of the charged and neutral systems (vertical detachment energies (VDE), the relative energies of the isomers of the neutral clusters, etc.). Energy calculations for charged systems require care in a supercell geometry with PBC, particularly where the energies of charged and neutral systems are compared. As in the case of the sulfur clusters, ${ }^{10}$ we extrapolate the Coulomb energy of the cluster in the above unit cell to give the corresponding energy for an isolated cluster with the same charge distribution.

We have performed additional all-electron calculations for isolated clusters ${ }^{33}$ to study the reliability of the pseudopotential and the extrapolation scheme for calculating the Coulomb energy. Following our findin that using a nonlocal approximation to $E_{\mathrm{xc}}$ gives rise to improved values of cohesive energies in phosphorus and arsenic clusters, ${ }^{22}$ we have performed similar tests here using the non-local modification to the exchange and correlation energies of Becke ${ }^{20}$ and Perdew, ${ }^{21}$ respectively. Vibration frequencies can be evaluated in two ways: In the MD/DF calculations we perturb equilibrium structures by random amounts and Fourier 
TABLE IV. Structure parameters for isomers of $\mathrm{P}_{n}^{-}, n=2-6$ with energies $\Delta E$ relative to the ground state $(\mathrm{eV})$. Bond lengths $d_{i j}$ in a.u., bond angles $\alpha_{i}$ in degrees. Additional labels refer to the figures

\begin{tabular}{|c|c|c|}
\hline Molecule & Symmetry & $\Delta E$ \\
\hline $\begin{array}{c}\mathrm{P}_{2}^{-} \\
d_{12}=3.72\end{array}$ & $D_{\infty h},{ }^{2} \Pi_{g}$ & 0.0 \\
\hline $\begin{aligned} & \mathrm{P}_{3}^{-} 7(\mathrm{a}) \\
d & =3.68\end{aligned}$ & $D_{\infty h},{ }^{1} \Sigma_{g}^{+}$ & 0.0 \\
\hline $\begin{array}{c}\mathrm{P}_{3}^{-} 7(\mathrm{~b}) \\
d=4.08, \alpha=60^{\circ}\end{array}$ & $D_{3 h},{ }^{3} A_{2}^{\prime}$ & 0.06 \\
\hline $\begin{array}{c}\mathrm{P}_{3}^{-} 7(\mathrm{c}) \\
d_{12,23}=3.90, \alpha_{123}=72.6^{\circ}\end{array}$ & $C_{2 v},{ }^{1} A_{1}$ & 0.27 \\
\hline $\begin{array}{c}\mathrm{P}_{4}^{-} 7(\mathrm{~d}) \\
d_{12}=4.18, d_{23}=4.09, \alpha_{21}\end{array}$ & $\begin{array}{c}C_{2 v} \\
C_{124}=77.1^{\circ}\end{array}$ & 0.0 \\
\hline $\begin{array}{c}\mathrm{P}_{5}^{-} 7(\mathrm{e}) \\
d=3.96, \alpha=108^{\circ}\end{array}$ & $D_{5 h}$ & 0.0 \\
\hline $\begin{array}{c}\mathrm{P}_{5}^{-} 7(\mathrm{f}) \\
d_{12}=4.28, d_{23}=4.18, d_{15}=\end{array}$ & $\begin{array}{c}C_{2 v} \\
=58.5^{\circ}, \alpha_{12}\end{array}$ & $\begin{array}{c}1.44 \\
54=77.5^{\circ}\end{array}$ \\
\hline $\begin{array}{c}\mathrm{P}_{6}^{-} 8(\mathrm{a}) \\
d_{12}=4.24, \quad d_{23}=4.18, \\
\alpha_{216}=108.2^{\circ}, \quad \alpha_{165}=104\end{array}$ & $\begin{array}{c}C_{2 v} \\
d_{56}=3.96\end{array}$ & $\begin{array}{c}0.0 \\
\alpha_{124}=90.1^{\circ},\end{array}$ \\
\hline $\begin{aligned} & \mathrm{P}_{6}^{-} 8(\mathrm{~b}) \\
& d_{12}=4.14, d_{16}=4.60\end{aligned}$ & $D_{3 h}$ & 0.30 \\
\hline \multicolumn{3}{|c|}{$d_{12}=4.06, d_{13}=4.18, d_{34}=4.45, \alpha_{123}=62.3^{\circ}, \alpha_{234}=99.8^{\circ}$} \\
\hline
\end{tabular}

analyze the subsequent motion, in the all-electron calculations the frequencies are determined from the spatial derivatives of the energy surfaces. In the case of $\mathrm{P}_{2}$ we have performed configuratio interaction (CI) calculations ${ }^{34}$ to clarify aspects of the multiplet structure of the neutral dimer.

Densities and spin densities used in the calculations are generated using occupation numbers compatible with a single determinant. If the states cannot be described in this way, as in the $3 s^{2} 3 p^{4}$ configuratio of the phosphorus anion, we adopt the approach of von Barth ${ }^{35}$ that is described in detail in Ref. 10. Calculations of excited states often require calculations for determinantal states that have holes below the Fermi energy. These have been performed by imposing constraints relating the occupation numbers and the order or symmetries of the orbitals.

\section{B. Results}

In this section we discuss the structures of phosphorus anions and the excitation energies to states of the neutral clusters. We focus on the vertical detachment energies (VDE), where the geometrical structure is unchanged, but adiabatic energy differences will be discussed where appropriate. Structural parameters of the anions are given in Tables IV-VI, and vibration frequencies in Table VII. Atomic coordinates are available from the authors. ${ }^{36}$
TABLE V. Structure parameters for isomers of $\mathrm{P}_{7}^{-}$and $\mathrm{P}_{8}^{-}$, with energies $\Delta E$ relative to the ground state $(\mathrm{eV})$. Bond lengths $d_{i j}$ in a.u., bond angles $\alpha_{i}$ in degrees. Additional labels refer to the figures

\begin{tabular}{|c|c|}
\hline Molecule & Symmetry \\
\hline $\begin{array}{c}\mathrm{P}_{7}^{-} 8(\mathrm{~d}) \\
d_{16}=4.28, \quad d_{12}=4.24, \\
\alpha_{137}=111.4^{\circ}, \alpha_{374}=10\end{array}$ & 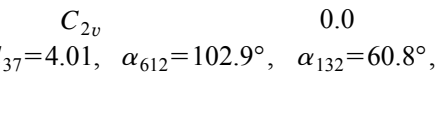 \\
\hline $\begin{array}{c}\mathrm{P}_{7}^{-} 8(\mathrm{e}) \\
d_{12}=4.15, \quad d_{17}=4.08 \\
\alpha_{216}=88.8^{\circ}, \alpha_{165}=89\end{array}$ & $\begin{array}{cc}C_{s} & 0.41 \\
d_{56}=4.18, & d_{46}=4.08, \\
0.8^{\circ}, \alpha_{654}=58.5^{\circ} & \alpha_{217}=83.9^{\circ},\end{array}$ \\
\hline $\begin{array}{l}\mathrm{P}_{7}^{-} 8(\mathrm{f}) \\
d_{16}=3.80, d_{13}=4.33,\end{array}$ & $\begin{array}{cc}C_{2 v} \\
=104.8^{\circ}, \alpha_{132}=92.5^{\circ}, \alpha_{137}=104.4^{\circ}\end{array}$ \\
\hline $\begin{array}{c}\mathrm{P}_{8}^{-} 9(\mathrm{a}) \\
d_{13}=4.26, d_{37}=4.02, c \\
\alpha_{134}=90.3^{\circ}, \quad \alpha_{137}=10 \\
\alpha_{346}=89.8^{\circ}, \quad \alpha_{456}=61 .\end{array}$ & $\begin{array}{c}C_{s} \\
=4.08, d_{25}=4.32, d_{45}=4.15, d_{46}=4.23, \\
100.1^{\circ}, \quad \alpha_{725}=100.2^{\circ}, \quad \alpha_{734}=96.7^{\circ},\end{array}$ \\
\hline $\begin{array}{c}\mathrm{P}_{8}^{-} 9(\mathrm{~b}) \\
d_{13}=4.33, \quad d_{78}=4.77, \\
\alpha_{273}=102.7^{\circ}\end{array}$ & $\begin{array}{cc}C_{2 v} & 0.23 \\
d_{25}=4.20, & \alpha_{456}=71.1^{\circ}, \quad \alpha_{643}=86.6^{\circ},\end{array}$ \\
\hline $\begin{array}{c}\mathrm{P}_{8}^{-} 9(\mathrm{c}) \\
d_{14}=4.07, \quad d_{34}=4.92 \\
\alpha_{145}=103.9^{\circ}, \alpha_{412}=9\end{array}$ & $\begin{array}{c}C_{2 v} \\
d_{36}=4.20, \quad \alpha_{345}=88.4^{\circ}, \quad \alpha_{341}=88.6^{\circ}, \\
1.2^{\circ}, \alpha_{432}=88.5^{\circ}, \alpha_{632}=91.8^{\circ}\end{array}$ \\
\hline $\begin{array}{l}\mathrm{P}_{8}^{-} 9(\mathrm{~d}) \\
l_{45}=4.10, d_{34}=4.86\end{array}$ & $D_{4 h}$ \\
\hline
\end{tabular}

\section{1. $\mathrm{P}_{1}^{-}$}

The energy differences between the ground state of the phosphorus anion and the low-lying states of the atom are calculated to be $0.85 \mathrm{eV}\left({ }^{4} S\right), 2.39 \mathrm{eV}\left({ }^{2} D\right)$ and $2.90 \mathrm{eV}\left({ }^{2} P\right)$. The measured electron affinit of phosphorus $(0.7465 \mathrm{eV})$ (Ref. 28) is one of the lowest of any element and reflect the half-full shell in the atom. All-electron calculations $^{33}$ give slightly smaller values than those found with the pseudopotential method, both with the LSD $(0.68$ $\mathrm{eV})$ and gradient-corrected $(0.66 \mathrm{eV})$ approximations to $E_{\mathrm{xc}}$.

TABLE VI. Structure parameters for isomers of $\mathrm{P}_{9}^{-}$, with energies $\Delta E$ relative to the ground state $(\mathrm{eV})$. Bond lengths $d_{i j}$ in a.u., bond angles $\alpha_{i}$ in degrees. Additional labels refer to the figures

\begin{tabular}{|c|c|c|}
\hline Molecule & Symmetry & $\Delta E$ \\
\hline \multicolumn{3}{|c|}{$\begin{array}{l}d_{34}=4.05, d_{45}=4.04, d_{56}=4.18, d_{67}=4.14, d_{78}=3.91, d_{16}=4.12, d_{12}=4.17, \\
d_{13}=4.31, \alpha_{345}=101.6^{\circ}\end{array}$} \\
\hline \multirow{2}{*}{\multicolumn{3}{|c|}{ 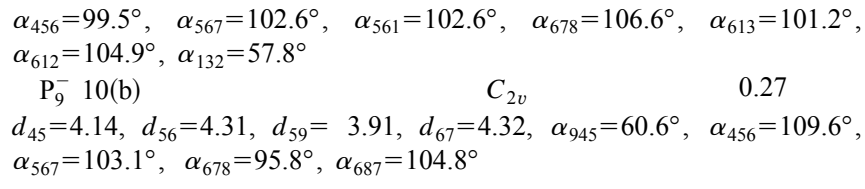 }} \\
\hline & & \\
\hline $\begin{array}{c}\mathrm{P}_{9}^{-} 10(\mathrm{c}) \\
d_{45}=4.03, \quad d_{56}=4 \\
\alpha_{456}=123.3^{\circ}, \alpha_{56}\end{array}$ & $\begin{array}{c}C_{2 v} \\
d_{67}=4.30, \\
5.8^{\circ}, \alpha_{678}=\end{array}$ & $\begin{array}{l}1.49 \\
345=110.0^{\circ}, \\
63.6^{\circ}\end{array}$ \\
\hline
\end{tabular}


TABLE VII. Vibration frequencies $\left(\omega_{e}, \mathrm{~cm}^{-1}\right)$ of selected phosphorus clusters.

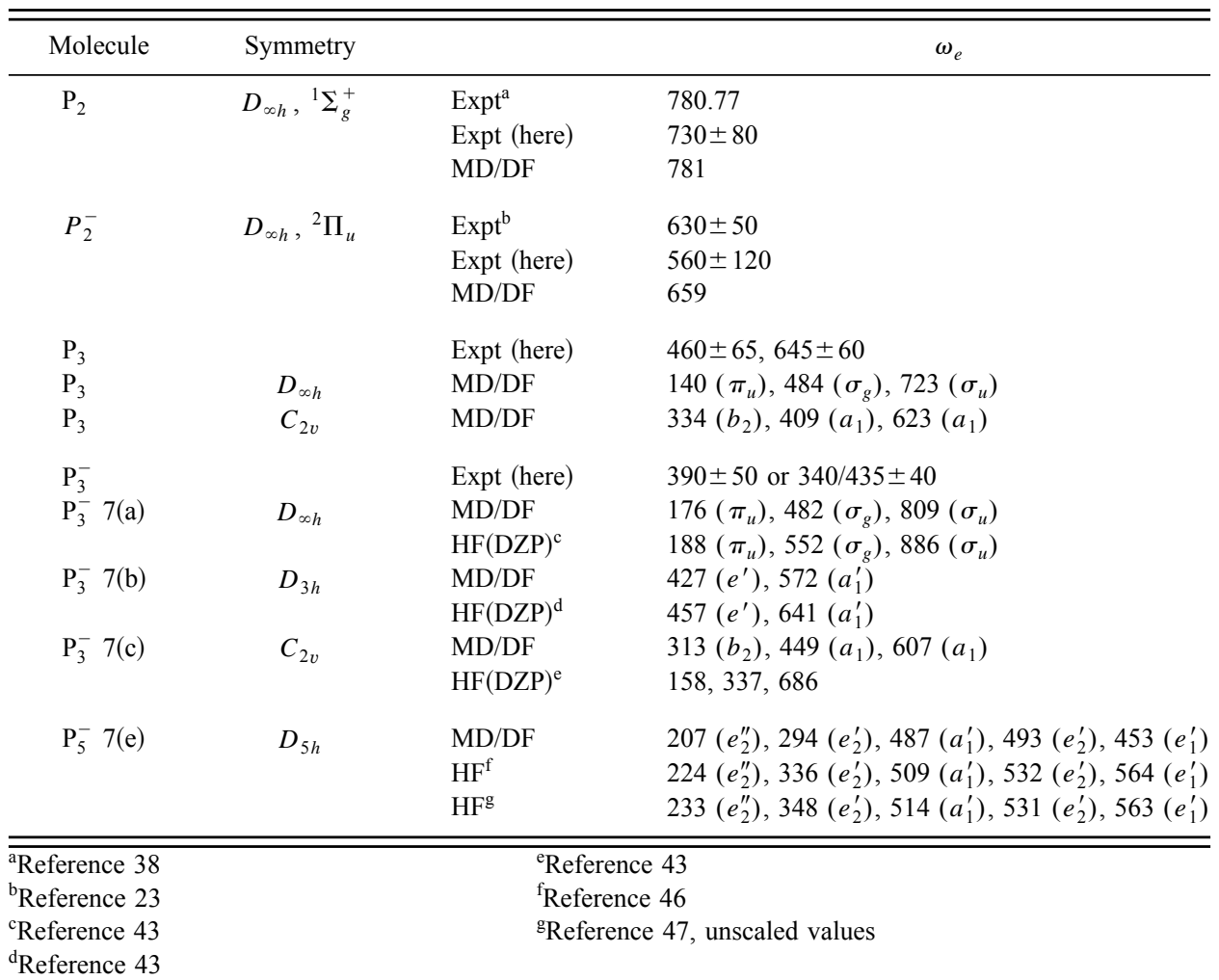

\section{2. $P_{2}^{-}$}

The calculated VDE of $\mathrm{P}_{2}^{-}(0.86 \mathrm{eV})$ corresponds to the excitation to the ground state of $\mathrm{P}_{2}$. A comparison of other features of the photoelectron spectra requires a description of low-lying excited states of the neutral dimer, and this causes some difficulties The energies of states derived from the ground state configuratio of $\mathrm{P}_{2}\left(\sigma_{g}^{2} \pi_{u}^{4},{ }^{1} \Sigma_{g}^{+}\right)$by the excitation $\sigma_{g} \rightarrow \pi_{g}\left(\sigma_{g} \pi_{u}^{4} \pi_{g} ;{ }^{3} \Pi_{g},{ }^{1} \Pi_{g}\right)$ are accessible to LSD calculations using the approach of von Barth, and we discuss these energies below. However, states arising from the excitations $\pi_{u} \rightarrow \pi_{g}\left(\sigma_{g}^{2} \pi_{u}^{3} \pi_{g} ;{ }^{3} \Sigma_{u}^{+},{ }^{3} \Sigma_{u}^{-},{ }^{3} \Delta_{u}\right.$, and related singlets) ${ }^{37}$ are indistinguishable in a LSD calculation, since the orbitals in the determinants required are the same. Information about these states can nevertheless be found from CI calculations and from experimental measurements on other group Va (group 15) dimers.

CI calculations ${ }^{34}$ have been performed for low-lying triplet states of $\mathrm{P}_{2}$ at the calculated equilibrium separation of the dimer anion. In these calculations it is possible to distinguish between states with "+" and "-" symmetry, i.e., the symmetry according to reflectio in a plane containing the molecular axis, and there are linear combinations of the $\pi_{g}$ - orbitals $\left(\pi_{x}, \pi_{y}\right)$ that have markedly different overlaps with the $\pi_{u}$-orbitals. Although the symmetries of the states could not be identifie unambiguously, the firs three triplets lie 2.64, 3.39, and $3.48 \mathrm{eV}$ above the ${ }^{1} \Sigma_{g}^{+}$state, in reasonable agreement with the measured energy differences $\left(T_{e}\right)$ for the (shorter) equilibrium separation of the ground state of $\mathrm{P}_{2}\left(2.33,3.53\right.$, and $3.50 \mathrm{eV}$ for ${ }^{3} \Sigma_{u}^{+},{ }^{3} \Sigma_{u}^{-}$, and ${ }^{3} \Pi_{g}$ states, respectively). ${ }^{38}$ The calculated vertical excitation energy (MD/DF) for the ${ }^{3} \Pi_{g}$ state is $4.22 \mathrm{eV}$, with an energy 3.30
eV above $\mathrm{P}_{2}\left({ }^{1} \Sigma_{g}^{+}\right)$at the energy minimum for $\mathrm{P}_{2}^{-}$. The narrow range of energies of the ${ }^{3} \Sigma_{u}^{-},{ }^{3} \Delta_{u}$, and ${ }^{3} \Pi_{g}$ states makes an assignment difficult Nevertheless, while the ${ }^{3} \Delta_{u}$ state has not yet been observed in $\mathrm{P}_{2}$, there is evidence that it lies between the ${ }^{3} \Sigma_{u}^{+}$and ${ }^{3} \Sigma_{u}^{-}$states in the other group Va (group 15) dimers $\mathrm{N}_{2},{ }^{38,39} \mathrm{As}_{2},{ }^{40}$ and $\mathrm{Bi}_{2}$ and $\mathrm{Sb}_{2} \cdot{ }^{24,41}$

\section{3. $P_{3}^{-}$}

The trimer anion $\mathrm{P}_{3}^{-}$has been investigated previously by Burdett and Marsden ${ }^{42}$ and Hamilton and Schaefer. ${ }^{43}$ Both sets of workers found three low-lying minima: an equilateral triangle $\left(D_{3 h},{ }^{3} A_{2}^{\prime}\right)$, a linear closed-shell singlet $\left(D_{\infty h},{ }^{1} \Sigma_{g}^{+}\right)$, and a bent $\left(C_{2 v}\right)$ triplet. The firs two were found to be so close in energy that a definit prediction of the ground state was not possible.

In the present $\mathrm{MD} / \mathrm{DF}$ calculations the linear structure has the lowest energy, but the $D_{3 h}$ and $C_{2 v}$ structures are only 0.06 and $0.27 \mathrm{eV}$ less stable. The vertical detachment energies of the three structures (Figs. 7(a)-(c)) show striking differences, however, being $3.00 \mathrm{eV}, 1.88 \mathrm{eV}$, and $1.73 \mathrm{eV}$, respectively. All-electron DF calculations with the LSD approximation are in remarkably good agreement, the energies relative to the linear structure being 0.03 and $0.25 \mathrm{eV}$, and the VDE values being 2.96, 1.84, and $1.64 \mathrm{eV}$, respectively. The inclusion of non-local modification to the energy functional reverses the energy ordering of the two most stable non-linear structures, with energies relative to the linear structure being -0.08 and $0.27 \mathrm{eV}$. CI calculations ${ }^{34}$ support the picture of two almost degenerate states (the $D_{3 h}$ structure is $0.03 \mathrm{eV}$ more stable) with the bent structure slightly $(0.28$ 

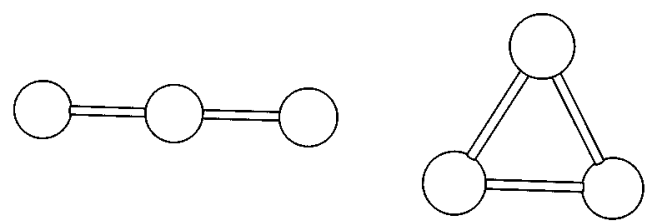

(a)

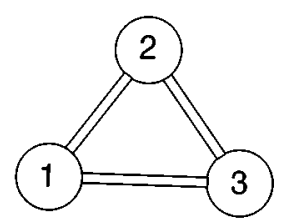

(c)

(d)
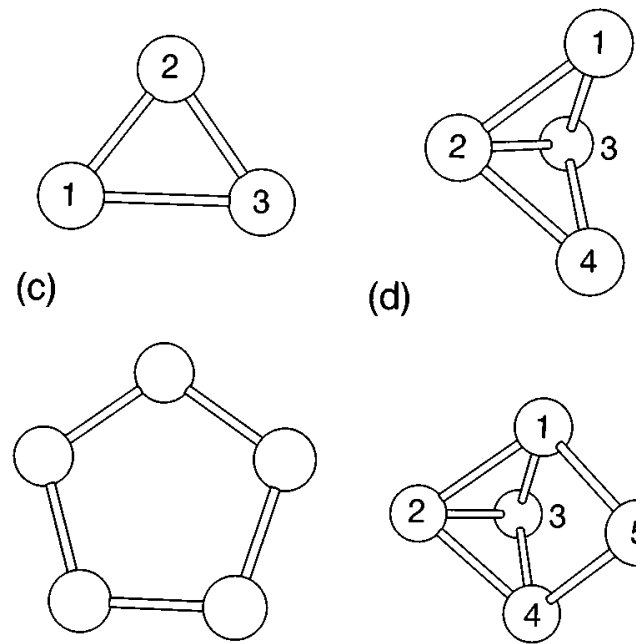

(e)

(f)

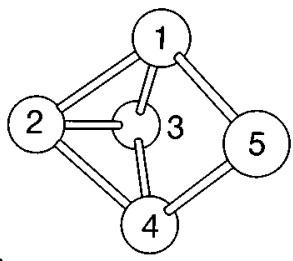

FIG. 7. Structures of (a-c) $\mathrm{P}_{3}^{-}$, (d) $\mathrm{P}_{4}^{-}$, and (e-f) $\mathrm{P}_{5}^{-}$.

$\mathrm{eV})$ higher. The calculated geometries are in good agreement with the earlier work. ${ }^{42,43}$

There are, of course, higher-lying states of $\mathrm{P}_{3}$ corresponding to each of the three structures found for $\mathrm{P}_{3}^{-}$. They will contribute to the measured intensity near peaks $(\mathrm{D}, \mathrm{E})$ in Fig. 5, but all occur at binding energies well above $3.0 \mathrm{eV}$. The lowest quartet state for the $D_{3 h}$ structure, for example, has a vertical excitation energy of $4.08 \mathrm{eV}$.

Vibration frequencies for $\mathrm{P}_{3}^{-}$have been calculated by Hamilton and Schaefer ${ }^{43}$ using the Hartree-Fock approach, and the results are compared with the present work in Table VII. Apart from the $C_{2 v}$ isomer, the results show the expected tendency of the HF method to overestimate vibration frequencies. All-electron density functional calculations give frequencies in very good agreement with the MD/DF calculations for all isomers, so that the very low frequencies found in Ref. 43 are difficul to understand.

\section{4. $P_{4}^{-}$}

The phosphorus tetramer has the familiar tetrahedral $\left(T_{d}\right)$ structure. The additional electron in the anion, however, results in a Jahn-Teller distortion that is so large that it is not meaningful to analyze the equilibrium structure (Fig. 7(d)) in terms of tetrahedral symmetry. The vertical detachment energies calculated for transitions to the lowest-lying singlet and triplet structures are 1.35 and $2.91 \mathrm{eV}$, respectively. Allelectron calculations give 1.55 and $2.80 \mathrm{eV}$ (LSD) and 1.54 and $2.62 \mathrm{eV}$, respectively. Relaxation of the neutral tetramer from the anionic structure to the tetrahedral form results in an energy lowering of $1.16 \mathrm{eV}$. The "roof" structure is also found to be the most stable in calculations for $\mathrm{Sb}_{4}^{-}$and $\mathrm{Bi}_{4}^{-} \cdot{ }^{25}$

\section{5. $P_{5}^{-}$}

The pentamer is an interesting case. The planar (cyclo- $\mathrm{P}_{5}$ ) anion has been stabilized as a ligand ${ }^{44}$ and prepared by reacting white phosphorus in solution with tetrahydrofuran. ${ }^{45}$ It has been calculated to be the most stable isomer in $\mathrm{Sb}_{5}^{-}$and $\mathrm{Bi}_{5}^{-} \cdot{ }^{25}$ Hartree-Fock calculations ${ }^{46,47}$ give a consistent picture of both geometry ( $\mathrm{P}-\mathrm{P}$ bond lengths of 3.955 and 3.959 a.u, respectively) and vibration frequencies (Table VII). Scherer and Brück ${ }^{44}$ noted that cyclo- $\mathrm{P}_{5}^{-}$ could possibly be formed from $\mathrm{P}_{3}^{-}$and $\mathrm{P}_{2}$, and Hamilton and Schaefer ${ }^{46}$ observed that the high frequency vibration observed by Baudler and coworkers ${ }^{45}$ could be due to the presence of $\mathrm{P}_{3}^{-}$. Calculations by Glukhotsev et al. ${ }^{48}$ indicate that the $D_{5 h}$ isomer of $\mathrm{P}_{5}^{-}$is stable with respect to both dissociation $\left(\mathrm{P}_{5}^{-} \rightarrow \mathrm{P}_{3}^{-}+\mathrm{P}_{2} ; \Delta E=71.6 \mathrm{kcal} / \mathrm{mol}\right)$ and disproportionation $\left(2 \mathrm{P}_{5}^{-} \rightarrow 2 \mathrm{P}_{3}^{-}+\mathrm{P}_{4}\left(T_{d}\right) ; \Delta E=116.4 \mathrm{kcal} / \mathrm{mol}\right.$. $)$

The present calculations lead to two low-lying isomers. The planar ring $\left(D_{5 h}\right.$, Fig. $\left.7(\mathrm{e})\right)$ has bonds of length 3.96 a.u. and is $1.44 \mathrm{eV}$ more stable than the structure (Fig. 7(f)) related to the most stable form found for the neutral pentamer. The planar ring is isovalent with the cyclopentadienyl anion $(\mathrm{CH})_{5}^{-}$and is an "aromatic" $6 \pi$-system. ${ }^{48}$ The relative stability of the pentagon is somewhat greater in all-electron calculations (LSD: $1.63 \mathrm{eV}$, NLSD: $1.76 \mathrm{eV}$ ). There is a striking difference between the vertical detachment energies of the two isomers of the anion $\left(D_{5 h}: 4.04 \mathrm{eV}, C_{2 v}: 2.08\right.$ $\mathrm{eV}$, with all-electron calculations giving very similar values). The calculated vibration frequencies (Table VII) are about $10 \%$ below the Hartree-Fock values, which are generally higher than experimental frequencies by approximately this amount. ${ }^{47}$

\section{6. $P_{6}^{-}$}

The most stable isomer in all the present calculations was the $C_{2 v}$ form (Fig. $8(\mathrm{a})$ ), which is $0.3-0.5 \mathrm{eV}$ more stable than the other isomers (Fig. 8(b), 8(c)). The vertical detachment energies are very similar for all three isomers, ranging from 2.3 to $2.7 \mathrm{eV}$ for transitions to the most stable (singlet) states of $\mathrm{P}_{6}$ and from 3.3 to $3.7 \mathrm{eV}$ for the lowest triplet of $\mathrm{P}_{6}$.

\section{7. $P_{7}^{-}$}

The main use of MD/DF calculations in the present work is as an effective generator and optimizer of cluster structures ("simulated annealing"). The process led in the heptamer $\mathrm{P}_{7}$ not only to low-lying isomers of the anions, but to a stable isomer of the neutral cluster-the $C_{2 v}$ structure shown in Fig. 8(d) - that had not been found in earlier work. ${ }^{16}$ The $\mathrm{MD} / \mathrm{DF}$ calculations indicate that this structure is slightly $(0.11 \mathrm{eV})$ more stable than the $C_{s}$ ("roof plus trimer") isomer, and this energy difference is very close to that found in all-electron calculations $(\sim 0.08 \mathrm{eV})$.

The anionic structure (Fig. 8(d)) is the most stable in all the present calculations, with a second isomer (Fig. 8(e)) 
(a)
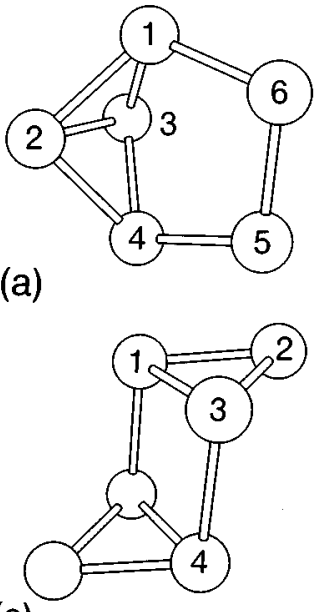

(c)

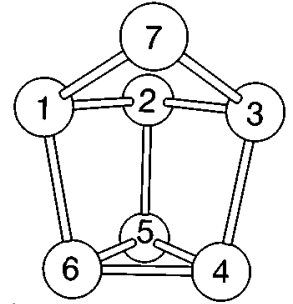

(e)

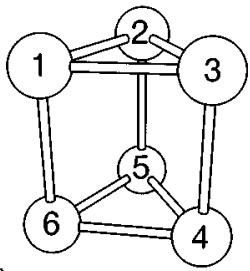

(b)

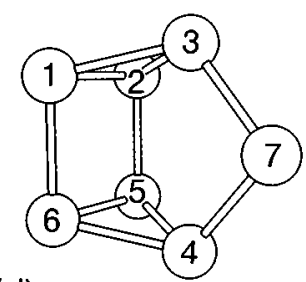

(d)

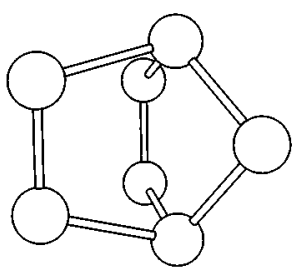

(f)

FIG. 8. Structures of (a-c) $\mathrm{P}_{6}^{-}$and (d-f) $\mathrm{P}_{7}^{-}$. Labels in (f) are as in (d).

$0.40-0.45 \mathrm{eV}$ higher. The open form (Fig. 8(f)) related to the former is a further $0.6 \mathrm{eV}$ higher. Structures Fig. 8(d), 8(e) are related to the prism structure of $\mathrm{P}_{6}$ by bridging bonds in and between the triangular faces, respectively. Comparison of Fig. 8(e) with the "roof plus trimer" structure of $\mathrm{P}_{7}$ (Fig. 5(b) of Ref. 16) shows that the effect of the additional charge in the anion is very large. The energy change in $\mathrm{P}_{7}$ between these two structures is correspondingly large $(0.68 \mathrm{eV})$.

The VDE of the three $\mathrm{P}_{7}^{-}$structures are very similar, ranging between $3.1-3.4 \mathrm{eV}$ in all the calculations. The fla energy surfaces of the heptamer, with or without charges, has interesting consequences. In the crystalline form, the $\mathrm{P}_{7}^{3-}$ ion has a $C_{3}$ cage structure related to that of $\mathrm{P}_{4} \mathrm{~S}_{3}$, but is flux ional in solution, i.e., it undergoes a pseudorotation that restores the original structure but with a different orientation. ${ }^{49}$ This process could be identifie in MD/DF simulations. ${ }^{17}$ The transition state involves a structure very similar to Fig. $8(\mathrm{~d})$. If we adopt this structure for the $\mathrm{P}_{7}^{+}$cluster, the twofold coordinated atom moves to a position above the center of a prism face with bonds of equal length to four atoms. If an electron is now added to the cluster, the structure can change to either Fig. 8(d) or 8(e), and we have observed both processes in MD/DF calculations. This "charge transfer induced isomerization" was also seen in our earlier work on $\mathrm{P}_{9} \cdot{ }^{18}$

The flexibilit of the heptamer isomers has stimulated an extensive search for possible stable structures. The starting structures for the simulations included two of interest: (a) the structure of $\mathrm{P}_{7}^{+}$mentioned above, with an atom centrally placed over a face of a prism, (b) the ground state structure $\left(C_{3}\right)$ of $\mathrm{P}_{7}^{3-}$. These structures relax rapidly to those of Fig. $8(\mathrm{e})$ and $8(\mathrm{~d})$, respectively.

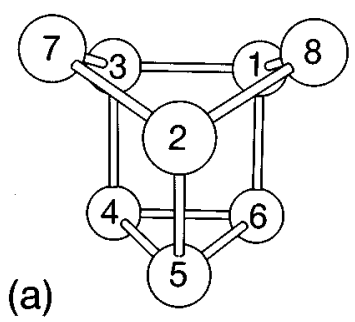

(b)

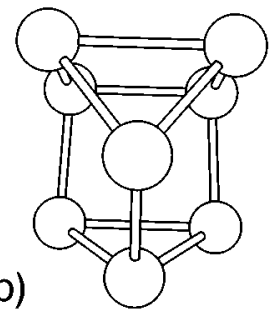

(c)

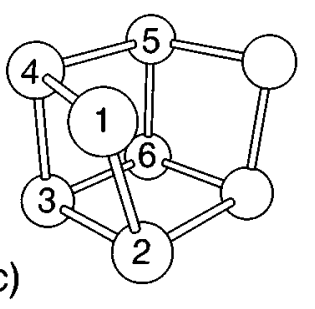

(d)

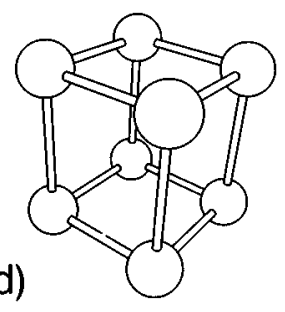

FIG. 9. Structures of $\mathrm{P}_{8}^{-}$. Labels in (b) and (d) are as in (a), (b), respectively.

\section{8. $P_{8}^{-}, P_{8}^{2-}$}

The predicted structures of the $\mathrm{P}_{8}$-isomers were perhaps the most unexpected results in the earlier study of neutral clusters. ${ }^{2}$ The cubic structure that had been favored by several authors proved to be much $(1.7 \mathrm{eV})$ less stable than the wedgelike $\left(C_{2 v}\right)$ analog of the cuneane form of $(\mathrm{CH})_{8}$. A third isomer $\left(D_{2 h}\right)$ had an energy between these two. Apart from the occurrence of $\mathrm{P}_{8}$-cages in crystalline phosphorus, ${ }^{50}$ we are unaware of any experimental information on the structure of the isolated clusters.

The MD/DF calculations predict several local minima in the energy surface. The two with the lowest energies are derived from the cuneane structure, with Fig. 9(a) being more stable by $0.22 \mathrm{eV}$. In the latter, there is a large expansion in two of the parallel bonds. This configuratio is unstable to annealing at $300 \mathrm{~K}$, with one of the long bonds breaking and the other contracting to give a structure with lower symmetry. A similar situation occurs in the structures related to a cube, where the more symmetrical structure (Fig. 9(d)) has four expanded bonds. The more stable of the pair (Fig. 9(c))—with energy $1.3 \mathrm{eV}$ above that of the most stable isomer-has one broken bond and seven bonds of length comparable with those in the cubic form of $\mathrm{P}_{8}$. Annealing from the $D_{2 h}$ structure of $\mathrm{P}_{8}{ }^{16}$ also results in structure Fig. 9(c).

All sets of calculations lead to VDE values that are significantl higher for the most stable isomer (Fig. 9(a)) than for all others. MD/DF calculations, for example, give $3.0 \mathrm{eV}$ for Fig. 9(a) and $2.5 \mathrm{eV}$ for Fig. 9(c). The MD/DF structure 9(b) does not correspond to an energy minimum in the allelectron calculations, and structure optimization leads directly to the most stable form 9(a).

Following the suggestion of Scherer, ${ }^{51}$ we have studied isomers of $\mathrm{P}_{8}^{2-}$, which is isovalent to the dianion of cyclooctatetraene $(\mathrm{CH})_{8}^{2-} \cdot{ }^{52}$ The latter has $10 \pi$-electrons and, in 


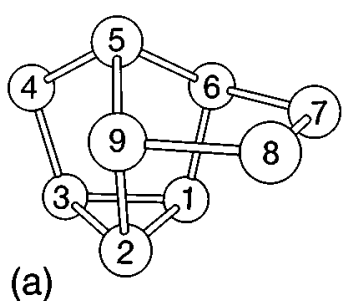

(b)

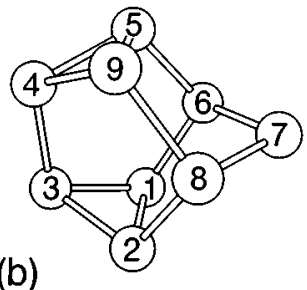

(c)

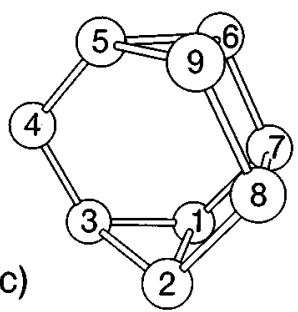

FIG. 10. Structures of $\mathrm{P}_{9}^{-}$.

accordance with the Hückel $(4 n+2)$ rule, is stable, planar and aromatic. We have found that $\mathrm{P}_{8}^{2-}$ also has a stable planar isomer, with all bond lengths and angles equal (3.96 a.u. - the same value as in planar $\mathrm{P}_{5}^{-}$- and $135^{\circ}$, respectively), but the energy is more than $1 \mathrm{eV}$ above the dianion structure related to 9(a). The planar ring isomer of the singly charged anion $\mathrm{P}_{8}^{-}$corresponds to a high-lying, shallow minimum in the energy surface and buckles on annealing at 200 $\mathrm{K}$.

\section{9. $P_{9}^{-}$}

All calculations predict that the most stable isomer of $\mathrm{P}_{9}^{-}$is the $C_{s}$ form shown in Fig. 10(a), which may be viewed as a dimer attached to the most stable form of $\mathrm{P}_{7}^{-}$. The $C_{2 v}$ unit 10(b) found in crystalline Hittorf's phosphorus, ${ }^{50}$ is only $\sim 0.2 \mathrm{eV}$ higher, and structure 10 (c) is more than $1 \mathrm{eV}$ less stable. The calculated VDE values are 3.3, 3.6 and 3.1 $\mathrm{eV}$, respectively.

\section{Trends}

The trends in the structures of the phosphorus cluster anions are reminiscent of of the neutral clusters. The structures are "three-dimensional" from $n=4$, and from $n=7$ they show distributions in bond lengths, bond angles, and dihedral angles that similar to those found in bulk phases of the element. Phosphorus atoms prefer three-fold coordination, although two-fold coordinated atoms generally have shorter bonds. The shortest bonds found were the multiple bonds occurring in the dimer and trimer. Bonds in rectangular structural units (bond angles $\sim 90^{\circ}$ ) are generally longer than those in triangular units, and the presence of rectangular units is energetically unfavorable. An example is the case of $\mathrm{P}_{9}^{-}$, where Fig. 10(c) is less stable than the otherwise similar structure 10(b). Rings with approximately pentagonal projections are found, and the most stable form of $\mathrm{P}_{8}^{-}$can be viewed as a "pentagon plus trimer" structure.

The anionic structures are generally more open than the corresponding neutral systems, and there are other differences arising from the different number of electrons. In $\mathrm{P}_{4}$, for example, the tetrahedral structure is stable, while $\mathrm{P}_{4}^{-}$undergoes a Jahn-Teller distortion. For $n=3,5$, on the other hand, the anion structures have higher symmetries $\left(D_{3 h}\right.$, $D_{5 h}$ ) than the Jahn-Teller distorted structures of $\mathrm{P}_{3}$ and $\mathrm{P}_{5}$. As in the case of the sulfur clusters, the presence of the additional electron in the anion can stabilize structures that are unstable in the neutral clusters. In $\mathrm{S}_{n}^{-}$these were chain structures that revert otherwise to the more stable rings. The vertical detachment energies in the former were larger than in the latter, where the most weakly bound electron occupied an orbital localized to one bond or atom. The differences in VDE are smaller in the phosphorus anions, but we note that $\mathrm{P}_{8}^{-}$has two local minima (Figs. 9(b), 9(d)) - corresponding to structures with two and four expanded bonds, respectively - that are less stable than the related structures (Figs. 9(a), 9(c)), where the additional charge is located in the region of one (broken) bond.

\section{COMPARISON OF THEORY AND EXPERIMENT}

The experimental results presented in Sec. II will now be compared with the results of the calculations described in Sec. III B. We shall discuss, in particular, the vibrational frequencies and the measurable energy differences, as well as the relative stabilities of the isomers.

\section{A. Vibration frequencies}

Vibrational frequencies could be resolved in the dimers and trimers, and the results are compared with calculated values (MD/DF) in Table VII. The calculations are in good agreement with all available experimental data for $\mathrm{P}_{2}$ and $\mathrm{P}_{2}^{-}$. The calculated values for the $a_{1}$ modes of the $C_{2 v}$ structure of $\mathrm{P}_{3}$ also agree well with the measured values for this molecule. We have noted above (Sec. II B 3.) that the measured spectrum of $\mathrm{P}_{3}^{-}$is consistent with two vibration frequencies (42 and $54 \mathrm{meV}, 340$ and $435 \mathrm{~cm}^{-1}$ ) or a single mode ( $48 \mathrm{meV}, 390 \mathrm{~cm}^{-1}$ ). The former are in fair agreement with the calculated frequencies for the $C_{2 v}$ structure, but the agreement is not as satisfactory as in the dimer and neutral trimer.

\section{B. Excitation energies}

The excitation energies provide valuable information, particularly in those cases where isomers of a cluster have distinctly different vertical detachment energies and/or adiabatic electron affinities It is important to note, however, that the excitations $\mathrm{P}_{n}^{-} \rightarrow \mathrm{P}_{n}$ are from the minimum of one energy surface to another surface where the energy can be a rapidly changing function of the geometry. This is particularly true in cases, such as the tetramer and the heptamer, where there is a large difference between the stable geometries of the anion and the neutral cluster. Small errors in the initial geometry can then to significan uncertainties in the excitation energy.

All calculations performed to date on $\mathrm{P}_{3}^{-}$give a consistent picture: The linear and equilateral triangular structure are virtually degenerate, and the bent $\left(C_{2 v}\right)$ form is only $\sim 0.2 \mathrm{eV}$ higher in energy. It is very likely that more than one 
isomer can be generated, depending on the experimental conditions. The spectra (Figs. 4,5) show fiv peaks (Table III). The calculated vertical excitation energies to the firs two states in $\mathrm{P}_{3}$ are $\left(1.73 \mathrm{eV}, 3.70 \mathrm{eV}\right.$ for $\left.C_{2 v}\right)$ and $(1.88 \mathrm{eV}, 4.08$ $\mathrm{eV}$ for $\left.D_{3 h}\right)$. In view of the uncertainties in both experiment and theory, either (or both) of these structures could be present. We note, however, the large gap in the excitation spectrum for both isomers, which means that the strong peak at $2.89 \mathrm{eV}$ must come from another structure. Since the linear isomer gives a vertical excitation at $3.00 \mathrm{eV}$, the measured binding energy curves are consistent with the existence of at least two isomers, one of which is linear. The photoelectron spectrum of $\mathrm{P}_{5}^{-}$at $h \nu=3.49 \mathrm{eV}$ was interpreted as fragmentation into $\mathrm{P}_{3}^{-}$and $\mathrm{P}_{2}$, with peak $\mathrm{C}$ arising from a different isomer than from peaks $\mathrm{A}$ and $\mathrm{B}$. This is consistent with the results of the calculations.

According to the above assignment, peak $\mathrm{C}$ in the spectrum of $\mathrm{P}_{3}^{-}$should exhibit fin structure corresponding to the vibrational modes of linear $\mathrm{P}_{3}$. The calculations indicate that the neutral and negatively charged trimers have the same bond lengths, so that a narrow peak should occur in the photoelectron spectrum. No fin structure is observed, and the peak is broader than expected. Both features are consistent with the thermal excitation of hot bands, since thermal excitation would result in the preferential excitation of low energy modes that are beyond the energy resolution of the apparatus. The relatively high vibrational temperature is consistent with the estimate in Sec. II B 2.

All features observed in the spectrum of $\mathrm{Sb}_{3}^{-}$have been assigned to emission from the $D_{3 h}$ isomer. ${ }^{24}$ In the absence of spin-orbit coupling, the present calculations, as well as those of Balasubramanian et al., ${ }^{53}$ predict a single vertical excitation energy to a ${ }^{2} E^{\prime \prime}$ state. This state, which undergoes a Jahn-Teller distortion to two nearby minima, would be split by spin-orbit coupling. It is presently not possible to say with certainty whether features $\mathrm{A}$ and $\mathrm{B}$ are due to the coexistence of $D_{3 h}$ and $C_{2 v}$ isomers or to transitions to two states of the former.

There are two pronounced peaks in the measured spectra of $\mathrm{P}_{4}^{-}$, at $1.35 \mathrm{eV}$ and $2.69 \mathrm{eV}$, both of which can be interpreted in terms of transitions from a "roof"-shaped isomer. The calculated excitation energies to the lowest singlet and firs triplet states of $\mathrm{P}_{4}$ are $1.35 \mathrm{eV}$ and $2.91 \mathrm{eV}$, respectively. The very broad firs peak indicates a large difference between the geometries of the most stable isomers of the anion and neutral clusters. The calculated adiabatic electron affin ity, the difference between the lowest energies of $\mathrm{P}_{4}^{-}$and $\mathrm{P}_{4}$ is only $0.19 \mathrm{eV}$, in satisfactory agreement with the onset in the measured spectra.

A single peak at $4.04 \mathrm{eV}$ dominates the spectrum of $\mathrm{P}_{5}^{-}$measured at $h \nu=4.66 \mathrm{eV}$. This is in excellent agreement with the calculated value for the planar pentagonal form (Fig. 7(e)). The $C_{2 v}$ isomer (Fig. 7(f)) related to the structure predicted to be the most stable in the neutral heptamer is much less stable and has a much lower excitation energy. There is no evidence that this isomer is generated by the PACIS.

The DF calculations indicate that the $C_{2 v}$ isomer of $\mathrm{P}_{6}^{-}$ (Fig. 8(a)) is the most stable. While the measured photoelec- tron spectra (peak positions given in Table I) are in reasonable agreement with the calculated values for the lowestlying states of $\mathrm{P}_{6}(2.29,3.33 \mathrm{eV})$, the other stable structures found give similar values $[2.44,3.70 \mathrm{eV}$ for Fig. 8 (b); 2.65, $3.43 \mathrm{eV}$ for Fig. 8(c)]. It is certainly possible that the source produces more than one isomer.

The measured intensities fall for clusters with more than six atoms, and relatively unstructured backgrounds become more apparent. The pronounced peak in the spectra of $\mathrm{P}_{7}^{-}$ (VDE $2.79 \mathrm{eV}$ ) is closest to the value calculated for the 8(d) structure $(3.11 \mathrm{eV})$, but the other two structures have excitation energies only $0.2 \mathrm{eV}$ higher. The structural relaxations on removal of an electron from structures 8(d) and 8(e) are, however, quite different. In the former, the anionic and neutral structures are very similar, the change in energy on relaxation is only $\sim 0.1 \mathrm{eV}$, and we expect a sharp rise in intensity to the maximum at the VDE. In the latter, the calculations predict a relaxation of $\sim 0.6 \mathrm{eV}$ and a much broader peak. Although the calculations predict that Fig. 8(d) is more than $0.4 \mathrm{eV}$ more stable than Fig. 8(e), the interpretation of the measured spectra is ambiguous, and it is possible that both isomers are present.

In $\mathrm{P}_{8}^{-}$, on the other hand, calculations of the vertical excitation energies are consistent with the results of total energy calculations, which favor the perturbed cage structure 9(a). The agreement between the measured VDE $(3.05 \mathrm{eV})$ and the calculated value $(3.02 \mathrm{eV})$ is very good, while the values for the other three isomers are much lower $(2.34-2.55$ $\mathrm{eV})$. The most stable isomers in $\mathrm{P}_{9}^{-}$have VDE values that are $3.60 \mathrm{eV}$ (Fig. 10(a)) and $3.28 \mathrm{eV}$ (Fig. 10(b)). The VDE for the latter, less stable structure is then in better agreement with the measured value $(3.11 \mathrm{eV})$. We note that the inclusion of non-local corrections to the exchange-correlation energy reduces the VDE values by $\sim 0.1-0.2 \mathrm{eV}$ in this anion.

\section{DISCUSSION AND CONCLUDING REMARKS}

We have performed a detailed study-both theoretical and experimental - of negatively charged and neutral phosphorus clusters $\mathrm{P}_{n}^{-}$, focusing on the geometries, excitation energies, and vibration frequencies. From an experimental point of view, phosphorus is a complicated material, and the samples we used were unstable in air. In spite of the special handling that this requires, it has been possible to generate and separate clusters up to $n=9$ and to perform photoelectron spectroscopy on them. The energy resolution in the spectra for $\mathrm{P}_{2}^{-}$and $\mathrm{P}_{3}^{-}$was sufficien to allow the determination of vibration frequencies for both.

The theoretical work used the combination of density functional calculations with molecular dynamics applied previously to neutral $\mathrm{P}_{n}$ clusters. The focus is twofold: (a) the calculation of the structures of isomers, i.e., locating the minima in the energy surfaces for each cluster anion, (b) determining the energy differences between the anions and states of the neutral clusters with the same geometry. The comparison with experiment concerns both such vertical excitation energies and the vibration frequencies. We have compared the results with previous work on phosphorus and related clusters. To examine the effects of approximations 
inherent in the $\mathrm{MD} / \mathrm{DF}$ approach, we have performed for all clusters all-electron calculations with and without non-local corrections to the exchange-correlation energy. The methods lead to very similar structures and excitation energies for phosphorus clusters. In the case of $\mathrm{P}_{2}$, we obtained useful complementary information with CI calculations.

The overall comparison between theory and experiment provides a consistent picture of the cluster isomers and their photoelectron spectroscopy. In those cases, such as $\mathrm{P}_{4}^{-}$, $\mathrm{P}_{5}^{-}$, and $\mathrm{P}_{8}^{-}$, where the energy calculations give an unambiguous prediction of the form of the most stable isomer, the calculated detachment energies are in satisfactory agreement with experiment. The measurements indicate that the most stable form of $\mathrm{P}_{8}^{-}$(with high probability $\mathrm{P}_{8}$ as well) has a "wedge" (cuneane) rather than a cubic structure. Calculations for the doubly charged anion $\mathrm{P}_{8}^{2-}$ show a stable planar $\left(D_{8 h}\right)$ isomer, analogous to the $10 \pi$-system $(\mathrm{CH})_{8}^{2-}$, with an energy more than $1 \mathrm{eV}$ above that of the isomer derived from the wedge form of $\mathrm{P}_{8}$. In $\mathrm{P}_{3}^{-}$, where the present and earlier calculations predict the existence of three isomers with different structures but very similar energies, we show that the source generates at least two isomers, one of them linear. Spectra for $\mathrm{P}_{5}^{-}$taken with $h \nu=3.49 \mathrm{eV}$ (below the electron affinit $4.04 \mathrm{eV}$ ) show fragmentation into $\mathrm{P}_{3}^{-}$and $\mathrm{P}_{2}$. We have noted above (Sec. III B 5) that there is evidence that the same process occurs in solutions containing the pentamer anion.

The situation in $\mathrm{P}_{7}^{-}$and $\mathrm{P}_{9}^{-}$is less definite The energies of the most stable isomers in these cases are only $\sim 0.2 \mathrm{eV}$ lower than the next most stable, and the vertical excitation energies in the $\mathrm{P}_{7}^{-}$isomers are similar. The VDE of the second most stable isomer of $\mathrm{P}_{9}^{-}$is in better agreement with the measured value than the value of the most stable isomer. The earlier work on sulfur clusters showed that the source can generate clusters that are not energetically the most stable.

This work has shown that the PACIS provides a flexibl method for generating and carrying out photoelectron spectroscopy on clusters of a very reactive material. Following previous work on metals (solid and liquid) and semiconductors, we believe that the source can provide interesting data for many other systems. The MD/DF approach should provide an ideal tool for analyzing these data.

\section{ACKNOWLEDGMENTS}

We thank numerous colleagues of our institute for helpful discussions, O.J. Scherer for comments on the manuscript, Hoechst AG (Hürth) for providing phosphorus samples, and the German Supercomputer Center (HLRZ) for a grant of CPU time on the Cray YMP8/864 in the Forschungszentrum Jülich.

${ }^{1}$ J. Donohue, The Structures of the Elements (Wiley, New York, 1974), Chap. 8.

${ }^{2}$ For a survey of earlier studies of phosphorus clusters, see R.O. Jones and D. Hohl, J. Chem. Phys. 92, 6710 (1990).

${ }^{3}$ T.P. Martin, Z. Phys. D 3, 211 (1986). Measurements on phosphorus clusters $\mathrm{P}_{n}$ have now been extended well beyond $n=6000$ (T.P. Martin and U. Näher, private communication).

${ }^{4}$ C. Bréchignac, Ph. Cahuzac, F. Carlier, M. de Frutos, J. Leygnier, and J. Ph. Roux, J. Chem. Phys. 102, 763 (1995).
${ }^{5}$ See, for example, O.J. Scherer, Angew. Chem. 102, 1137 (1990); Angew. Chem. Int. Ed. Engl. 29, 1104 (1990); O.J. Scherer, K. Pfeiffer, and G. Wolmershäuser, Chem. Ber. 125, 2367 (1992).

${ }^{6}$ M. Baudler and K. Glinka, Chem. Rev. 93, 1623 (1993).

${ }^{7}$ H.G. von Schnering and W. Hönle, Chem. Rev. 88, 243 (1988).

${ }^{8}$ M. Häser, J. Am. Chem. Soc. 116, 6925 (1994); S. Böcker and M. Häser, Z. Anorg. Allg. Chem. 621, 258 (1995).

${ }^{9}$ C.Y. Cha, G. Ganteför, and W. Eberhardt, Rev. Sci. Instrum. 63, 5661 (1992). See also H. Handschuh, G. Ganteför, and W. Eberhardt, ibid. 66, 3838 (1995).

${ }^{10}$ S. Hunsicker, R.O. Jones, and G. Ganteför, J. Chem. Phys. 102, 5917 (1995); G. Ganteför, S. Hunsicker, and R.O. Jones, Chem. Phys. Lett. 236, 43 (1995).

${ }^{11}$ R.D. Mead, A.E. Stephens, and W.C. Lineberger, in Gas Phase Ion Chemistry, edited by M.T. Bowers (Academic, Orlando, 1984), p. 213, and references therein.

${ }^{12}$ See, for example, R.O. Jones and O. Gunnarsson, Rev. Mod. Phys. 61, 689 (1989); R.M. Dreizler and E.K.U. Gross, Density Functional Theory (Springer, Berlin, 1990).

${ }^{13}$ R. Car and M. Parrinello, Phys. Rev. Lett. 55, 2471 (1985).

${ }^{14}$ D. Hohl and R.O. Jones, Phys. Rev. B 45, 8995 (1992) (amorphous P).

${ }^{15}$ D. Hohl and R.O. Jones, Phys. Rev. B 50, 17047 (1994) (liquid P).

${ }^{16}$ R.O. Jones and D. Hohl, J. Chem. Phys. 92, 6710 (1990) (clusters up to $\mathrm{P}_{8}$ ).

${ }^{17}$ G. Seifert and R.O. Jones, J. Chem. Phys. 96, 2951 (1992) (isomerization of $\mathrm{P}_{7}^{3-}$ ).

${ }^{18}$ R.O. Jones and G. Seifert, J. Chem. Phys. 96, 7564 (1992) (clusters up to $\mathrm{P}_{11}$ ).

${ }^{19}$ M. Häser, U. Schneider, and R. Ahlrichs, J. Am. Chem. Soc. 114, 9551 (1992).

${ }^{20}$ A.D. Becke, Phys. Rev. A 38, 3098 (1988).

${ }^{21}$ J.P. Perdew, Phys. Rev. B 33, 8822 (1986); 34, 7406(E) (1987).

${ }^{22}$ P. Ballone and R.O. Jones, J. Chem. Phys. 100, 4941 (1994).

${ }^{23}$ J.T. Snodgrass, J.V. Coe, C.B. Freidhoff, K.M. McHugh, and K.H. Bowen, Chem. Phys. Lett. 122, 352 (1985).

${ }^{24}$ M.L. Polak, J. Ho, G. Gerber, and W.C. Lineberger, J. Chem. Phys. 95, 3053 (1991) $\left[\mathrm{Bi}_{n}\right]$; M.L. Polak, G. Gerber, J. Ho, and W.C. Lineberger, ibid. 97, 8990 (1992) [ $\left.\mathrm{Sb}_{n}\right]$.

${ }^{25}$ M. Gausa, R. Kaschner, H.O. Lutz, G. Seifert, and K.H. Meiwes-Broer, Chem. Phys. Lett. 230, 99 (1994); M. Gausa, R. Kaschner, G. Seifert, J.H. Faehrmann, H.O. Lutz, and K.H. Meiwes-Broer (to be published).

${ }^{26}$ G. Ganteför, H.R. Siekmann, H.O. Lutz, and K.H. Meiwes-Broer, Chem. Phys. Lett. 165, 293 (1990); H.R. Siekmann, C. Lüder, J. Faehrmann, H.O. Lutz, and K.H. Meiwes-Broer, Z. Phys. D 20, 417 (1991).

${ }^{27}$ E.C. Honea, M.L. Homer, J.L. Persson, and R.L. Whetten, Chem. Phys. Lett. 171, 147 (1990)

${ }^{28}$ H. Hotop and W.C. Lineberger, J. Phys. Chem. Ref. Data 14, 731 (1985).

${ }^{29}$ W.C. Martin, R. Zalubas, and A. Musgrove, J. Phys. Chem. Ref. Data 14, 751 (1985).

${ }^{30}$ The spin-orbit splitting in the ground states of the isoelectronic PS and $\mathrm{SiCl}$ molecules are approximately 40 and $26 \mathrm{meV}$, respectively, and in the ground state $\left({ }^{2} \Pi_{u}\right)$ of $\mathrm{P}_{2}^{+} 32 \mathrm{meV}$. See Ref. 38 .

${ }^{31}$ J. Ho, K.M. Ervin, and W.C. Lineberger, J. Chem. Phys. 93, 6987 (1990).

${ }^{32}$ G. Bachelet, D.R. Hamann, and M. Schlüter, Phys. Rev. B 26, 4199 (1982).

${ }^{33}$ DGAUSS program from the UniChem package of Cray Research, Inc. The calculations used a triple zeta basis with polarization functions (TZP) and the A1 auxiliary basis.

${ }^{34}$ GAUSSIAN 92/DFT, Revision G.2, M.J. Frisch et al., Gaussian, Inc., Pittsburgh, Pennsylvania (1993). The calculations were quadratic CI calculations with single and double excitations and a correction for triples [UQCISD(T)] and used the 6-311G(d) basis.

${ }^{35}$ U. von Barth, Phys. Rev. A 2, 1693 (1979).

${ }^{36}$ Contact R.O. Jones (Internet: r.jones@kfa-juelich.de)

${ }^{37}$ G. Herzberg, Molecular Spectra and Molecular Structure. I. Spectra of Diatomic Molecules (Van Nostrand, Princeton, 1950).

${ }^{38}$ K.P. Huber and G. Herzberg, Molecular Spectra and Molecular Structure. IV. Constants of Diatomic Molecules (Van Nostrand Reinhold, New York, 1979).

${ }^{39}$ R.R. Laher and F.R. Gilmore, J. Phys. Chem. Ref. Data 20, 685 (1991).

${ }^{40}$ L.A. Heimbrook, N. Chestnoy, M. Rasanen, G.P. Schwartz, and V.E. Bondybey, J. Chem. Phys. 83, 6091 (1985).

${ }^{41}$ K. Balasubramanian and J. Li, J. Mol. Spectrosc. 135, 169 (1989).

${ }^{42}$ J.K. Burdett and C.J. Marsden, New J. Chem. 12, 797 (1988). 
${ }^{43}$ T.P. Hamilton and H.F. Schaefer III, Chem. Phys. Lett. 166, 303 (1990).

${ }^{44}$ O.J. Scherer and T. Brück, Angew. Chem. 99, 59 (1987), Angew. Chem. Int. Ed. Engl. 26, 59 (1987).

${ }^{45}$ M. Baudler and D. Ouzounis, Z. Naturforsch. Teil B 44, 381 (1989), and references therein.

${ }^{46}$ T.P. Hamilton and H.F. Schaefer III, Angew. Chem. 101, 500 (1989).

${ }^{47}$ R. Janoschek, Chem. Ber. 122, 2121 (1989).
${ }^{48}$ M.N. Glukhovtsev and P. v.R. Schleyer, J. Phys. Chem. 97, 8200 (1993).

${ }^{49}$ M. Baudler, H. Ternberger, W. Faber, and W. Hahn, Z. Naturforsch. Teil B 34, 1690 (1979)

${ }^{50}$ H. Thurn and H. Krebs, Acta Crystallogr. B 25, 125 (1969).

${ }^{51}$ O.J. Scherer (private communication).

${ }^{52}$ L.A. Paquette, Tetrahedron 31, 2855 (1975).

${ }^{53}$ K. Balasubramanian, K. Sumathi, and D. Dai, J. Chem. Phys. 95, 3494 (1991). 